\begin{tabular}{|c|c|}
\hline AUG $151996 \%$, ENGINEERING DATA TRANSMITTAL & 1. EDT NO 612675 \\
\hline
\end{tabular}

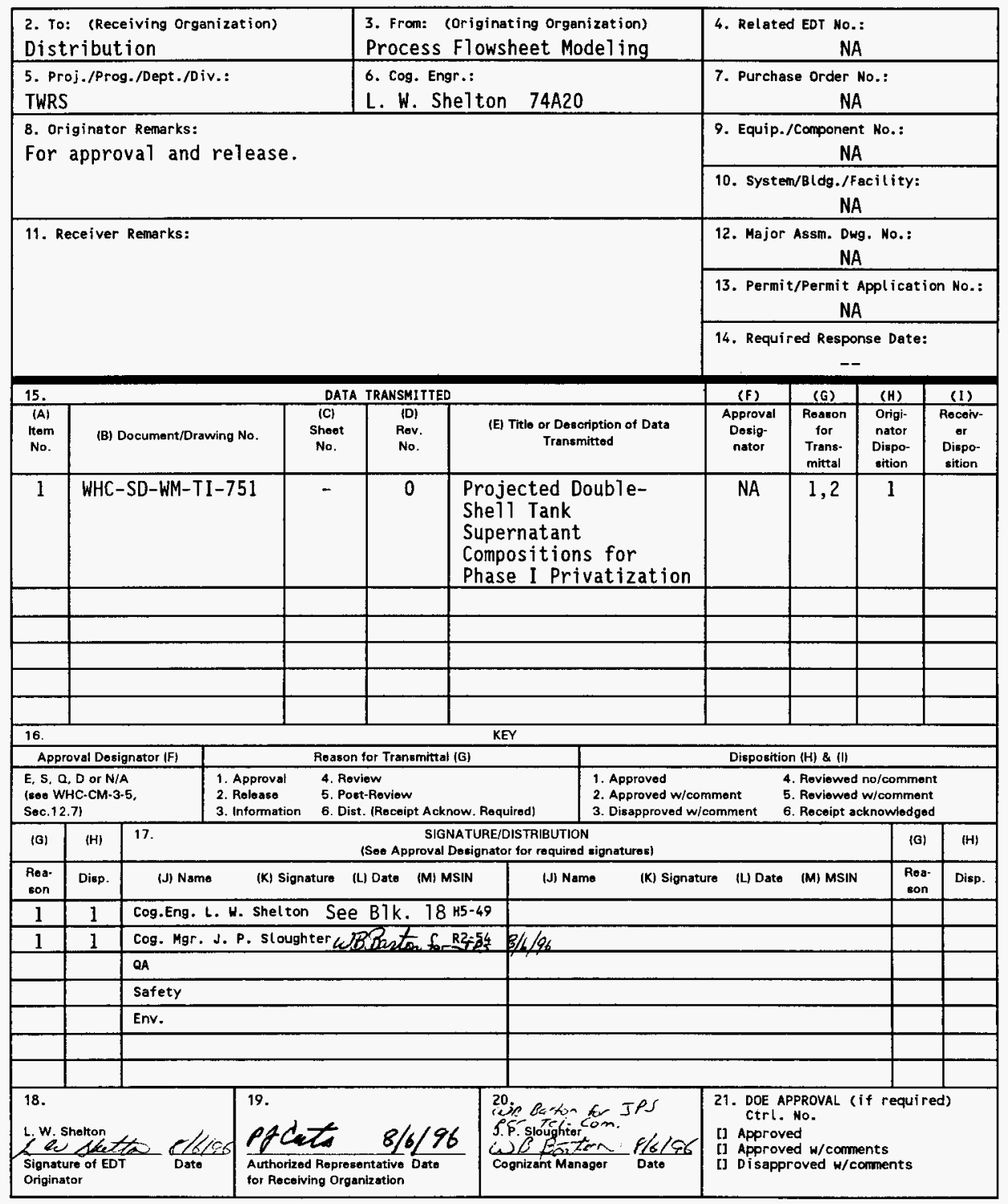




\section{Projected Double-Shell Tank Supernatant Compositions for Phase I Privatization}

L. W. Shelton

Westinghouse Hanford Company, Richland, WA 99352

U.S. Department of Energy Contract DE-AC06-87RL10930

EDT/ECN: 612675 UC: 721

Org Code: 74A20 Charge Code: 06129

B\&R Code: EW3130010 Total Pages: 49

Key Words: compositions, double-shell tank, low-level waste, feed staging, Phase I, privatization

Abstract: A set of projected double-shell tank compositions is developed to support the low-level waste feed staging $\mathrm{plan}$ used to support Phase I privatization efforts.

TRADEMARK DISCLAIMER. Reference herein to any specific commercial product, process, or service by trade name, trademark, manufacturer, or otherwise, does not necessarily constitute or imoly its endorsement, recommendation, or favoring by the United States Goverment or any agency thereof or its contractors or subcontractors.

Printed in the United States of America. To obtain copies of this document, contact: WHC/BCS Document Control Services, P.O. Box 1970, Mailstop H6-08, Richland WA 99352, Phone (509) 372-2420; Fax (509) 376-4989.
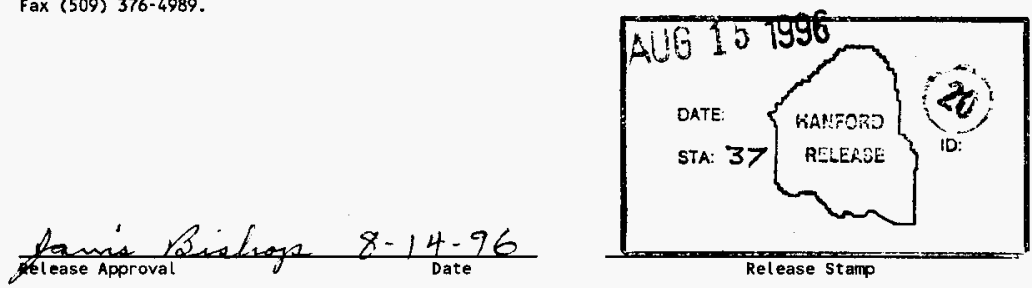

\section{Approved for Public Release}


WHC-SD-WM-TI-751

Revision 0

\section{PROJECTED DOUBLE-SHELL TANK SUPERNATANT COMPOSITIONS \\ FOR PHASE I PRIVATIZATION}

July 1996

L. W. Shelton

Westinghouse Hanford Company

Richland, Washington

Prepared for

U.S. Department of Energy

Richland, Washington 


\section{WHC-SD-WM-TI-751}

Revision 0

This page intentionally left blank. 
WHC-SD-WM-TI-751

Revision 0

CONTENTS

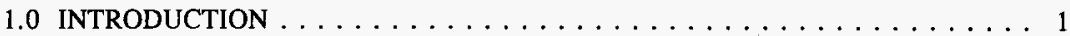

1.1 BACKGROUND $\ldots \ldots \ldots \ldots \ldots \ldots \ldots \ldots \ldots \ldots \ldots \ldots \ldots \ldots \ldots \ldots$

1.2 PURPOSE AND SCOPE $\ldots \ldots \ldots \ldots \ldots \ldots \ldots \ldots \ldots \ldots$

2.0 SUMMARY AND CONCLUSIONS $\ldots \ldots \ldots \ldots \ldots \ldots \ldots \ldots \ldots$

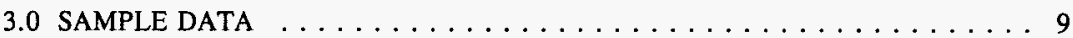

4.0 PROJECTING DOUBLE-SHELL TANK SUPERNATANT COMPOSITIONS $\ldots .$.

4.1 USING HISTORICAL TRANSACTION RECORDS TO PROJECT

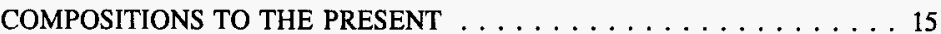

4.2 USING THE OPERATIONAL WASTE VOLUME PROJECTION TO

ESTIMATE FUTURE COMPOSITIONS . . . . . . . . . . . 23

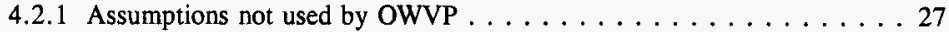

5.0 SUMMARY OF TRANSACTIONS AND QUALITY OF PROJECTIONS $\ldots \ldots 29$

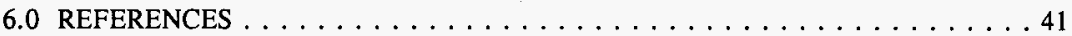

\section{LIST OF TABLES}

2-1. Projected Double-Shell Tank Supernate Compositions for Fiscal Year $2002 \ldots .44$

3-1. Analytical Results for Double-Shell Tank Supernatants $\ldots \ldots \ldots \ldots$

4-1. Historical Transactions for $241-\mathrm{AW}-105 . \ldots \ldots \ldots \ldots \ldots$

4-2. Facility Waste and Saltwell Liquid Composition Estimates . . . . . . . . . 19

4-3. Summary of Historical Transactions for Double-Shell Tanks Supernatants . . . . 21

4-4. Operational Waste Volume Projections Flush Volume Assumptions $\ldots \ldots \ldots 23$

4-5. Summary of Assumed Future Transactions for

Double-Shell Tanks Supernatants . . . . . . . . . . . . . . 24

5-1. Quality of Projected Supernate Composition Estimates for Double-Shell Tanks . . 39 
WHC-SD-WM-TI-751

Revision 0

\section{LIST OF TERMS}

$\begin{array}{ll}\text { CC } & \text { Concentrated complexant } \\ \text { CP } & \text { Concentrated phosphate } \\ \text { CSWL } & \text { Complexed saltwelllLiquid } \\ \text { DN } & \text { Dilute non-complexed } \\ \text { DSS } & \text { Double-shell slurry } \\ \text { DSSF } & \text { Double-shell slurry feed } \\ \text { DST } & \text { Double-shell tank } \\ \text { Evap Bott } & \text { Evaporator bottoms } \\ \text { Evap } & \text { Evaporator } \\ \text { FY } & \text { Fiscal year } \\ \text { GA } & \text { Gain } \\ \text { HLW } & \text { High-level waste } \\ \text { INST } & \text { Instrument recalibration } \\ \text { LLW } & \text { Low-level waste } \\ \text { LO } & \text { Loss } \\ \text { M\&I } & \text { Management and Integration } \\ \text { NCAW } & \text { Neutralized current acid waste } \\ \text { NCRW } & \text { Neutralized cladding removal waste } \\ \text { NCSWL } & \text { Non-complexed saltwell liquid } \\ \text { OWVP } & \text { Operational Waste Volume Projection } \\ \text { PFP } & \text { Plutonium Finishing Plant } \\ \text { PUREX } & \text { Plutonium-uranium extraction } \\ \text { PXMCS } & \text { Plutonium-uranium extraction miscellaneous wastes } \\ \text { SST } & \text { Single-shell tank } \\ \text { SWL } & \text { Saltwell liquid } \\ \text { TOC } & \text { Total Organic Carbon } \\ \text { TRU } & \text { Transuranic } \\ \text { TWRS } & \text { Tank Waste Remediation System } \\ \text { WHC } & \text { Westinghouse Hanford Company } \\ & \end{array}$


WHC-SD-WM-TI-751

Revision 0

PROJECTED DOUBLE-SHELL TANK SUPERNATANT

COMPOSITIONS FOR PHASE I PRIVATIZATION

\subsection{INTRODUCTION}

\subsection{BACKGROUND}

In 1991, the U.S. Department of Energy (DOE) established the Tank Waste Remediation System (TWRS) whose mission is "to store, treat, and immobilize highly radioactive Hanford Waste... in a safe, environmentally sound, and cost-effective manner ${ }^{n}$ (WHC 1995). Currently, there are approximately $1.35 \mathrm{E}+05 \mathrm{~m}^{3}(35.8 \mathrm{Mgal})$ of waste in 149 single-shell tanks (SSTs) and $7.42 \mathrm{E}+04 \mathrm{~m}^{3}(19.6 \mathrm{Mgal})$ of waste in 28 double-shell tanks (DSTs).

A new strategy for remediation of Hanford Site tank waste, commonly called "privatization," is being pursued by the U.S. Department of Energy, Richland Operations Office (RL). Privatization involves the hiring of private contractors to treat and immobilize tank waste on a pay-for-product basis. During Phase I, the technical, regulatory, and financial viability of the privatization concept will be demonstrated by pretreating and immobilizing a portion of the supernate stored in the DST system into a low-activity waste (LAW) product.

This proof-of-concept demonstration has been scheduled by RL to begin on June 1, 2002 (DOE-RL 1995). The Management and Integration (M\&I) contractor is required to provide two private contractors with appropriate quantities of feed of a specified composition on or before the June 1, 2002, startup date (Certa et al. 1996a).

To support Phase I privatization, Westinghouse Hanford Company (WHC), is preparing a Low-Level Waste (LLW) Feed Staging Plan (Certa et al. 1996b) that will develop a strategy for the delivery of feed to the private contractors. 


\section{WHC-SD-WM-TI-751}

\section{Revision 0}

\subsection{PURPOSE AND SCOPE}

The LLW Feed Staging Plan must use as a basis composition estimates for DST supernatants that will exist near the start of staging activities. The purpose of this report is to develop a set of DST supernate compositions projected to fiscal year (FY) 2002 to support the LLW Feed Staging Plan.

The scope of this report includes projecting DST supernate inventories to the beginning of staging activities and assessing the quality of the projections. 
WHC-SD-WM-TI-751

Revision 0

\subsection{SUMMARY AND CONCLUSIONS}

A set of projected DST supernatant composition estimates projected to FY 2002 has been derived from sample results, historical transaction sheets, waste profile data sheets, and operational waste volume projections. The projected composition estimates for selected components are shown in Table 2-1. An estimate of the quality of projection is provided at the end of Table 2-1. The 241-SY-101-SOL, and 241-SY-103-SOL designations indicate what is expected to be the soluble portion of the entire waste content of these two tanks. These are included because it is unlikely that only the supernatant portions of these tanks will be retrieved separately. The contents of 241-SY-101 are being continuously mixed by a mixer pump and the contents of 241-SY-103 are expected to be continuously mixed.

The series of steps performed to arrive at projected compositions for the DSTs is summarized as follows:

- A set of initial DST supernate compositions was derived, beginning with the most reliable sample data and supernate volumes at the time of sampling.

- Historical transaction records that chronicle transfers of waste into and out of the DST system, in addition to transfers made between DSTs, were used to project supernate compositions to July 1995. Compositions for wastes entering the DST system from facilities were taken from waste profile data sheets that are used to assess waste compatibility before transfer. Compositions for saltwell liquids were taken from Sederburg (1995).

- The Operational Waste Volume Projections (OWVP) (Strode 1995) were used in combination with composition estimates for facility wastes and saltwell liquids from SST stabilization efforts to project DST supernate compositions to FY 2002.

The reliability of these compositions as indicated by the quality of the projection depend primarily on the number of transactions involved with a given tank, the accuracy of average waste compositions reported by the various facilities, and the future validity of the assumptions used in the OWVP. Although the projections in the OWVP represent the most comprehensive source of information regarding future DST system activity, some of the assumptions will likely change as events unfold. This report recognizes that several OWVP assumptions pertaining to aging waste consolidation will have to be modified in light of recent studies that suggest that the assumptions in the OWVP are not practical. In fact, the most recent revision of the OWVP, still in draft form, contains a number of new assumptions concerning waste transfers that are not reflected in this report. Equipment failures, revision of volume estimates, waste incompatibilities and other safety concerns are a few examples of how OWVP assumptions may have to change in the future. 
Table 2-1. Projected Double-Shell Tank Supernate Compositions for Fiscal Year 2002. (4 Sheets)

\begin{tabular}{|c|c|c|c|c|c|c|c|c|c|}
\hline Supernstant & $\begin{array}{c}\mathrm{Al}(\mathrm{OH}) 4 \\
(\mathrm{M})\end{array}$ & $\begin{array}{c}\mathrm{B} \theta+2 \\
(\mathrm{M})\end{array}$ & $\begin{array}{c}\mathrm{Ca}+2 \\
(\mathrm{M})\end{array}$ & $\begin{array}{c}C d+2 \\
(M)\end{array}$ & $\begin{array}{c}\mathrm{Cr}(\mathrm{OH}) 4- \\
(\mathrm{M})\end{array}$ & $\begin{array}{c}\mathrm{Fe}+3 \\
(\mathrm{M})\end{array}$ & $\begin{array}{c}\mathrm{Hg}+2 \\
(\mathrm{M})\end{array}$ & $\begin{array}{l}\mathrm{K}+ \\
(\mathrm{M})\end{array}$ & $\begin{array}{c}\mathbf{L a}+3 \\
(\mathrm{M})\end{array}$ \\
\hline 241-AN-101 & $9.05 \mathrm{E}-02$ & $2.22 \mathrm{E}-05$ & $2.77 \mathrm{E}-03$ & $1.54 \mathrm{E}-04$ & $6.55 \mathrm{E}-03$ & $2.12 \mathrm{E}-03$ & $3.54 \mathrm{E}-06$ & $1.09 \mathrm{E}-02$ & $1.31 \mathrm{E}-04$ \\
\hline 241-AN-102 & $5.39 \mathrm{E}-01$ & & $1.08 \mathrm{E}-02$ & & $5.39 \mathrm{E}-03$ & & & $9.80 \mathrm{E}-02$ & \\
\hline 241-AN-103 & $2.13 E+00$ & & $2.16 \mathrm{E}-03$ & $1.42 \mathrm{E}-04$ & $1.63 \mathrm{E}-02$ & $1.26 \mathrm{E}-03$ & $7.98 \mathrm{E}-05$ & $3.89 \mathrm{E}-01$ & \\
\hline 241-AN-104 & $1.39 \mathrm{E}+00$ & & & & $1.30 \mathrm{E}-02$ & $2.00 \mathrm{E}-04$ & & $1.83 \mathrm{E}-01$ & \\
\hline 241-AN-105 & $1.74 \mathrm{E}+00$ & & & & $1.30 \mathrm{E}-02$ & $2.00 \mathrm{E}-04$ & & $1.57 \mathrm{E}-01$ & \\
\hline 241-AN-106 & $7.40 \mathrm{E}-01$ & $4.08 \mathrm{E}-07$ & $3.06 \mathrm{E}-06$ & $1.22 \mathrm{E}-06$ & 2.51E-05 & $3.19 \mathrm{E}-05$ & $1.57 \mathrm{E}-09$ & 3.75E-05 & \\
\hline 241-AN-107 & 4.22E-02 & & $1.30 \mathrm{E}-02$ & & $3.10 \mathrm{E}-03$ & 2.55E-02 & 0.00 & $5.14 \mathrm{E}-02$ & \\
\hline 241-AP-101 & $3.17 \mathrm{E}-01$ & $1.67 \mathrm{E}-05$ & $2.46 \mathrm{E}-03$ & 1.67E-05 & $2.60 \mathrm{E}-03$ & $4.61 E-04$ & 1.33E-08 & 5.33E-01 & $1.01 \mathrm{E}-04$ \\
\hline 241-AP-102 & $4.30 \mathrm{E}-01$ & $2.07 \mathrm{E}-06$ & & $1.31 \mathrm{E}-05$ & $1.19 \mathrm{E}-02$ & $6.82 \mathrm{E}-05$ & & $3.30 \mathrm{E}-02$ & \\
\hline 241-AP-103 & $9.11 \mathrm{E}-03$ & & & $9.87 \mathrm{E}-07$ & 8.44E-05 & $2.20 \mathrm{E}-05$ & $2.49 \mathrm{E}-08$ & $3.89 \mathrm{E}-02$ & \\
\hline 241-AP-104 & $2.83 \mathrm{E}-01$ & $1.37 \mathrm{E}-15$ & $5.74 \mathrm{E}-11$ & $4.96 \mathrm{E}-16$ & $1.47 \mathrm{E}-10$ & 4.62E-05 & & $4.90 \mathrm{E}-08$ & \\
\hline 241-AP-105 & $6.59 \mathrm{E}-01$ & $4.81 \mathrm{E}-07$ & $6.21 \mathrm{E}-05$ & $3.59 \mathrm{E}-08$ & $1.49 \mathrm{E}-05$ & $2.28 \mathrm{E}-06$ & & $1.90 \mathrm{E}-03$ & $4.96 \mathrm{E}-06$ \\
\hline 241-AP-106 & $1.23 \mathrm{E}+00$ & $2.26 \mathrm{E}-07$ & $2.84 \mathrm{E}-04$ & $2.41 \mathrm{E}-11$ & $2.69 \mathrm{E}-04$ & $9.00 \mathrm{E}-05$ & & $9.89 \mathrm{E}-02$ & 0.00 \\
\hline 241-AP-107 & $7.01 \mathrm{E}-01$ & $1.27 \mathrm{E}-05$ & 1. $65 \mathrm{E}-03$ & $9.46 \mathrm{E}-07$ & $3.99 \mathrm{E}-04$ & $6.01 \mathrm{E}-05$ & & $5.20 \mathrm{E}-02$ & $1.31 \mathrm{E}-04$ \\
\hline 241-AP-108 & 8.06E-03 & $1.16 \mathrm{E}-06$ & $1.66 \mathrm{E}-05$ & $5.16 \mathrm{E}-05$ & $1.04 \mathrm{E}-04$ & $1.25 \mathrm{E}-04$ & & $2.58 \mathrm{E}-02$ & \\
\hline 241-AW-101 & $1.03 \mathrm{E}+00$ & & $8.26 \mathrm{E}-04$ & & $3.10 \mathrm{E}-03$ & 0.00 & & $1.07 \mathrm{E}+00$ & \\
\hline \multicolumn{10}{|l|}{$241-\mathrm{AW}-102^{\mathrm{a}}$} \\
\hline 241-AW-103 & $1.54 \mathrm{E}-03$ & 4.08E-06 & $1.54 \mathrm{E}-04$ & & $1.40 \mathrm{E}-03$ & 0.00 & & $5.22 \mathrm{E}-01$ & \\
\hline 241-AW-104 & $9.77 \mathrm{E}-03$ & $2.03 E-08$ & $6.68 \mathrm{E}-07$ & & $1.31 \mathrm{E}-07$ & $5.44 \mathrm{E}-06$ & & $2.76 \mathrm{E}-06$ & \\
\hline 241-AW-105 & $1.99 \mathrm{E}-04$ & $6.94 \mathrm{E}-09$ & $8.26 \mathrm{E}-06$ & & $7.04 \mathrm{E}-06$ & $8.89 \mathrm{E}-04$ & & $2.63 \mathrm{E}-03$ & \\
\hline \multicolumn{10}{|l|}{ 241-AW-106 } \\
\hline 241-AY-101 & $2.61 \mathrm{E}-01$ & $3.64 \mathrm{E}-07$ & $4.52 \mathrm{E}-05$ & & $2.05 \mathrm{E}-02$ & 1.55E-06 & & $7.68 \mathrm{E}-02$ & $3.84 \mathrm{E}-06$ \\
\hline 241-AY-102 & $6.68 \mathrm{E}-05$ & $4.95 \mathrm{E}-08$ & $6.20 \mathrm{E}-06$ & $3.52 \mathrm{E}-07$ & $2.86 \mathrm{E}-06$ & $4.85 \mathrm{E}-06$ & $8.16 \mathrm{E}-09$ & 0.00 & $2.98 \mathrm{E}-07$ \\
\hline 241-AZ-101 & $8.19 \mathrm{E}-02$ & $3.77 \mathrm{E}-07$ & $2.17 \mathrm{E}-05$ & $2.85 \mathrm{E}-07$ & $3.82 \mathrm{E}-03$ & $4.72 \mathrm{E}-06$ & & $2.34 \mathrm{E}-02$ & $7.15 \mathrm{E}-07$ \\
\hline 241-AZ-102 & $5.88 \mathrm{E}-04$ & $2.14 \mathrm{E}-07$ & $2.55 \mathrm{E}-05$ & $2,94 \mathrm{E}-07$ & $1.39 \mathrm{E}-03$ & $5.00 \mathrm{E}-06$ & & $6.11 E-04$ & $4.30 \mathrm{E}-07$ \\
\hline 241-SY-101 & $1.58 \mathrm{E}+00$ & & $1.87 \mathrm{E}-02$ & & $1.56 \mathrm{E}-03$ & $3.51 \mathrm{E}-04$ & & $1.27 E-01$ & \\
\hline $\begin{array}{l}241-\text { SY- }^{\circ} \\
101-\text { SOL }^{b}\end{array}$ & $9.30 \mathrm{E}-01$ & & $1.24 \mathrm{E}-02$ & & $3.08 \mathrm{E}-03$ & $3.48 \mathrm{E}-04$ & & $6.59 \mathrm{E}-02$ & \\
\hline 241-SY-102 & 8.95E-02 & & 1. $34 \mathrm{E}-12$ & & $3.43 \mathrm{E}-12$ & $6.50 \mathrm{E}-05$ & & 1.15E-09 & \\
\hline 241-SY-103 & $8.17 \mathrm{E}-01$ & & $7.97 \mathrm{E}-04$ & & $1.71 \mathrm{E}-04$ & 0.00 & & $2.71 \mathrm{E}-02$ & 0.00 \\
\hline $\begin{array}{l}241-S Y-103- \\
\text { SOL }^{b}\end{array}$ & $1.17 \mathrm{E}+\infty$ & $4.83 \mathrm{E}-05$ & $4.77 \mathrm{E}-03$ & & $9.07 \mathrm{E}-02$ & $2.24 \mathrm{E}-02$ & & $7.35 \mathrm{E}-02$ & \\
\hline
\end{tabular}

241-AW-102 and 241-AW-106 are not included. They are the evaporator feed and receipt tanks, respectively and their projected compositions are too difficult to determine.

241-SY-101-SOL and 241-SY-103-SOL represent the soluble portion of the entire waste content of 241-SY-101 and 241-SY-103. 
Table 2-1. Projected Double-Shell Tank Supernate Compositions for Fiscal Year 2002. (4 Sheets)

\begin{tabular}{|c|c|c|c|c|c|c|c|c|c|}
\hline Supernatant & $\begin{array}{r}\mathrm{N}_{\mathrm{a}}+ \\
(\mathrm{M})\end{array}$ & $\begin{array}{c}\mathrm{Ni}+3 \\
(\mathrm{M})\end{array}$ & $\begin{array}{c}\mathrm{Pb}+4 \\
(\mathrm{M})\end{array}$ & $\underset{(g / l)}{U}$ & $\begin{array}{c}\cos -2 \\
(M)\end{array}$ & $\begin{array}{l}\mathrm{CL} \\
(\mathrm{M}) \\
\end{array}$ & $\begin{array}{l}\text { F- } \\
\text { (M) }\end{array}$ & $\begin{array}{c}504-2 \\
(\mathrm{M})\end{array}$ & $\begin{array}{c}\text { No3- } \\
\text { (M) }\end{array}$ \\
\hline 241-AN-101 & $2.75 E+00$ & 4.13E-06 & $1.16 \mathrm{E}-04$ & $2.87 \mathrm{E}-01$ & $1.60 \mathrm{E}-01$ & $1.04 \mathrm{E}-02$ & $3.34 \mathrm{E}-02$ & $4.96 \mathrm{E}-02$ & 2.91E-01 \\
\hline $241-\mathrm{AN}-102$ & $1.14 \mathrm{E}+01$ & $5.88 \mathrm{E}-03$ & 0.00 & 0.00 & $1.10 \mathrm{E}+00$ & $1.08 \mathrm{E}-01$ & $1.08 \mathrm{E}-01$ & $1.47 \mathrm{E}-01$ & $3.50 \mathrm{E}+00$ \\
\hline 241-AN-103 & $1.46 \mathrm{E}+01$ & $4.09 \mathrm{E}-04$ & $3.47 \mathrm{E}-04$ & $5.18 \mathrm{E}-04$ & 1.49E-01 & $2.71 \mathrm{E}-01$ & $3.87 \mathrm{E}-02$ & $1.67 \mathrm{E}-02$ & $2.58 \mathrm{E}+00$ \\
\hline 241-AN-104 & $1.20 \mathrm{E}+01$ & & & & $4.72 \mathrm{E}-01$ & $2.12 \mathrm{E}-01$ & & $6.82 \mathrm{E}-02$ & $3.10 \mathrm{E}+00$ \\
\hline 241-AN-105 & $1.20 \mathrm{E}+01$ & & & & $3.63 \mathrm{E}-01$ & $2.40 \mathrm{E}-01$ & & $6.82 \mathrm{E}-02$ & $3.12 \mathrm{E}+00$ \\
\hline 241-AN-106 & $1.61 \mathrm{E}+01$ & & 3.02E-06 & 2.13E-04 & $9.80 \mathrm{E}-01$ & $4.52 \mathrm{E}-04$ & $1.56 \mathrm{E}-01$ & $1.19 \mathrm{E}-01$ & $4.58 E+00$ \\
\hline 241-AN-107 & $9.19 \mathrm{E}+00$ & $7.27 \mathrm{E}-03$ & $1.51 \mathrm{E}-03$ & $2.13 \mathrm{E}-01$ & $1.19 \mathrm{E}+00$ & 8.29E-02 & & $1.35 \mathrm{E}-01$ & $3.63 E+00$ \\
\hline 241-AP-101 & $6.06 \mathrm{E}+00$ & $3.64 \mathrm{E}-04$ & $1.72 \mathrm{E}-05$ & $3.49 \mathrm{E}-02$ & 3.35E-01 & $4.92 \mathrm{E}-02$ & 2.09E-01 & 4.27E-02 & 2.13E +00 \\
\hline 241-AP-102 & $4.43 \mathrm{E}+00$ & $4.53 \mathrm{E}-04$ & $1.59 \mathrm{E}-05$ & 3.52E-03 & $4.47 \mathrm{E}-01$ & 8.18E-02 & 0.00 & $4.70 \mathrm{E}-02$ & $1.26 \mathrm{E}+00$ \\
\hline 241-AP-103 & $2.31 \mathrm{E}-01$ & & & $3.69 \mathrm{E}-03$ & $4.28 \mathrm{E}-02$ & $1.40 \mathrm{E}-03$ & $6: 79 \mathrm{E}-03$ & $3.64 \mathrm{E}-03$ & $6.52 \mathrm{E}-02$ \\
\hline 241-AP-104 & $2.43 \mathrm{E}+00$ & $9.50 \mathrm{E}-16$ & $5.38 \mathrm{E}-15$ & $2.06 \mathrm{E}-03$ & $1.24 \mathrm{E}-01$ & $8.19 \mathrm{E}-02$ & $1.04 \mathrm{E}-01$ & $1.62 \mathrm{E}-02$ & $6.83 \mathrm{E}-01$ \\
\hline 241-AP-105 & $1.44 \mathrm{E}+01$ & 1.24E-05 & $5.93 \mathrm{E}-08$ & 3.17E-04 & $8.76 \mathrm{E}-01$ & $2.94 \mathrm{E}-04$ & 1.32E-01 & $1.06 \mathrm{E}-01$ & $4.08 \mathrm{E}+00$ \\
\hline 241-AP-106 & $1.06 \mathrm{E}+01$ & 4.62E-11 & $2.62 \mathrm{E}-10$ & $6.31 \mathrm{E}-03$ & $5.40 \mathrm{E}-01$ & $3.45 \mathrm{E}-01$ & $6.23 \mathrm{E}-01$ & $6.91 \mathrm{E}-02$ & $3.08 \mathrm{E}+00$ \\
\hline $241-\mathrm{AP}-107$ & $1.55 \mathrm{E}+01$ & $3.27 \mathrm{E}-04$ & $1.6 \mathrm{tE}-06$ & $8.40 \mathrm{E}-03$ & $9.74 \mathrm{E}-01$ & $7.77 \mathrm{E}-03$ & $1.48 \mathrm{E}-01$ & $1.25 \mathrm{E}-01$ & $4.45 \mathrm{E}+00$ \\
\hline 241-AP-108 & $2.53 \mathrm{E}-01$ & $6.82 \mathrm{E}-06$ & 0.00 & $3.85 \mathrm{E}-03$ & $4.29 \mathrm{E}-02$ & $1.64 \mathrm{E}-03$ & $1.89 \mathrm{E}-02$ & $1.55 \mathrm{E}-03$ & $6.91 \mathrm{E}-02$ \\
\hline 241-AW-101 & $1.00 \mathrm{E}+01$ & & $1.46 \mathrm{E}-03$ & $2.24 \mathrm{E}-01$ & & $1.46 \mathrm{E}-01$ & & $1.07 \mathrm{E}-02$ & $3.45 \mathrm{E}+00$ \\
\hline \multicolumn{10}{|l|}{ 241-AW-102 } \\
\hline 241-AW-103 & 8.52E-01 & 5.05E-05 & & $9.87 \mathrm{E}-03$ & $7.68 \mathrm{E}-02$ & $3.81 \mathrm{E}-03$ & $9.16 \mathrm{E}-01$ & $5.86 \mathrm{E}-04$ & $5.66 \mathrm{E}-02$ \\
\hline 241-AW-104 & $1.97 \mathrm{E}-01$ & 2.07E-09 & $2.99 \mathrm{E}-09$ & $2.17 \mathrm{E}-02$ & 8.05E-03 & $5.24 \mathrm{E}-03$ & $3.56 \mathrm{E}-04$ & $2.64 \mathrm{E}-04$ & $6.25 \mathrm{E}-02$ \\
\hline 241-AW-105 & $9.47 \mathrm{E}-02$ & & & $1.99 \mathrm{E}-02$ & 3.57E-04 & $1.16 \mathrm{E}-03$ & $4.98 \mathrm{E}-03$ & $2.43 \mathrm{E}-03$ & $3.18 \mathrm{E}-01$ \\
\hline \multicolumn{10}{|l|}{ 241-AW-106 } \\
\hline 241-AY-101 & $4.53 \mathrm{E}+00$ & 9.27E-06 & & $9.46 \mathrm{E}-01$ & $6.56 \mathrm{E}-01$ & $3.76 \mathrm{E}-03$ & $9.46 \mathrm{E}-02$ & $2.30 \mathrm{E}-01$ & $1.04 E+00$ \\
\hline 241-AY-102 & $1.26 \mathrm{E}-01$ & & $2.59 \mathrm{E}-07$ & $1.25 \mathrm{E}-05$ & $1.03 \mathrm{E}-04$ & $5.56 \mathrm{E}-06$ & 1.56E-05 & 2.69E-05 & $1.11 \mathbf{E}-02$ \\
\hline 241-AZ-101 & $9.96 \mathrm{E}-01$ & 1.18E-06 & $2.51 \mathrm{E}-06$ & 4.47E-03 & $7.11 \mathrm{E}-02$ & $6.59 \mathrm{E}-04$ & $2.25 \mathrm{E}-02$ & $2.16 \mathrm{E}-02$ & $2.00 \mathrm{E}-01$ \\
\hline 241-AZ-102 & 2.87E-01 & 1.09E-06 & $6.36 \mathrm{E}-07$ & $9.89 \mathrm{E}-02$ & $2.72 \mathrm{E}-02$ & & 3.45E-03 & $1.02 \mathrm{E}-02$ & $3.30 \mathrm{E}-02$ \\
\hline 241-SY-101 & $1.17 \mathrm{E}+01$ & $8.86 \mathrm{E}-04$ & & $3.39 \mathrm{E}-03$ & & $3.89 \mathrm{E}-01$ & & $1.43 \mathrm{E}-02$ & $3.68 \mathrm{E}+00$ \\
\hline $\begin{array}{l}241-\mathrm{SY}- \\
101-\mathrm{SOL}\end{array}$ & $7.00 \mathrm{E}+00$ & $3.93 \mathrm{E}-04$ & & & & $1.84 \mathrm{E}-01$ & $4.96 \mathrm{E}-03$ & $3.59 \mathrm{E}-02$ & $5.80 \mathrm{E}-01$ \\
\hline $241-S Y-102$ & 8.72E-01 & & & $2.90 \mathrm{E}-03$ & $4.36 \mathrm{E}-02$ & $2.87 \mathrm{E}-02$ & $3.18 \mathrm{E}-02$ & $6.44 \mathrm{E}-03$ & $2.20 \mathrm{E}-01$ \\
\hline $241-S Y-103$ & $1.12 \mathrm{E}+01$ & $2.24 \mathrm{E}-04$ & & $7.64 \mathrm{E}-04$ & 5.32E-01 & 8.85E-02 & 8.02E-02 & $6.42 \mathrm{E}-02$ & $3.26 \mathrm{E}+00$ \\
\hline $\begin{array}{c}241-\text { SY-103. } \\
\text { SOL }\end{array}$ & $7.00 \mathrm{E}+00$ & $1.06 \mathrm{E}-03$ & & $3.60 \mathrm{E}-01$ & $3.53 \mathrm{E}-01$ & $1.99 \mathrm{E}-01$ & $3.95 \mathrm{E}-02$ & $3.96 \mathrm{E}-02$ & $1.79 \mathrm{E}-01$ \\
\hline
\end{tabular}

241-AW-102 and 241-AW-106 are not included. They are the evaporator food and receipt tanks, respectively and their projected compositions are too difficult to determine.

241-SY-101-SOL and 241-SY-103-SOL represent the soluble portion of the entire waste content of 241-SY-101 and 241-SY-103. 
WHC-SD-WM-TI-751

Revision 0

Table 2-1. Projected Double-Shell Tank Supernate Compositions for

Fiscal Year 2002. (4 Sheets)

\begin{tabular}{|c|c|c|c|c|c|c|c|c|c|}
\hline Supernatant & $\begin{array}{c}\text { NO2- } \\
\text { (M) }\end{array}$ & $\begin{array}{l}\text { PO4-3 } \\
\text { (M) }\end{array}$ & $\begin{array}{l}\text { OH- } \\
\text { (M) }\end{array}$ & $\begin{array}{l}\text { TOC } \\
(\mathrm{g} / 1)\end{array}$ & $\begin{array}{c}{ }^{90} \mathrm{Sr} \\
(\mathrm{Ci} / \mathrm{L})\end{array}$ & $\begin{array}{c}{ }^{99} \mathrm{Tc} \\
(\mathrm{Ci} / \mathrm{L})\end{array}$ & $\begin{array}{l}{ }^{137} \mathrm{Cs} \\
(\mathrm{Ci} / \mathrm{L})\end{array}$ & $\begin{array}{l}{ }^{239} \mathrm{Pu} \\
(\mathrm{Ci} / \mathrm{L})\end{array}$ & $\begin{array}{l}{ }^{240} \mathrm{Pu} \\
(\mathrm{Ci} / \mathrm{L})\end{array}$ \\
\hline 241-AN-101 & $2.59 \mathrm{E}-01$ & $1.22 \mathrm{E}-01$ & $1.64 \mathrm{E}+00$ & $2.14 \mathrm{E}+00$ & $3.81 \mathrm{E}-02$ & $9.49 \mathrm{E}-05$ & 3.05E-01 & $2.47 \mathrm{E}-05$ & $1.07 \mathrm{E}-06$ \\
\hline $241-A N-102$ & $1.78 \mathrm{E}+00$ & $5.10 \mathrm{E}-02$ & $6.18 \mathrm{E}-0 \mathrm{t}$ & $2.58 \mathrm{E}+01$ & $5.61 \mathrm{E}-02$ & $3.04 \mathrm{E}-04$ & $1.86 \mathrm{E}-01$ & $5.77 \mathrm{E}-05$ & 1.44E-05 \\
\hline 241-AN-103 & $3.00 \mathrm{E}+00$ & $9.77 \mathrm{E}-03$ & $5.74 E+\infty$ & $7.36 \mathrm{E}+\infty$ & $8.42 \mathrm{E}-03$ & $1.70 \mathrm{E}-04$ & $4.98 \mathrm{E}-01$ & $1.90 \mathrm{E}-06$ & $4.75 \mathrm{E}-07$ \\
\hline 241-AN-104 & $1.92 \mathrm{E}+00$ & $2.94 \mathrm{E}-02$ & $4.09 \mathrm{E}+00$ & $4.61 \mathrm{E}+\infty$ & $6.97 \mathrm{E}-03$ & $1.31 \mathrm{E}-04$ & $5.45 \mathrm{E}-01$ & $8.32 \mathrm{E}-06$ & 2.08E-06 \\
\hline 241-AN-105 & $2.61 \mathrm{E}+00$ & $2.00 \mathrm{E}-02$ & $3.64 \mathrm{E}+00$ & $3.88 \mathrm{E}+\infty$ & $2.29 \mathrm{E}-03$ & $1.31 \mathrm{E}-04$ & 3.64E-01 & 8.32E-06 & 2.08E-06 \\
\hline 241-AN-106 & $2.56 \mathrm{E}+00$ & $7.83 \mathrm{E}-02$ & $5.92 \mathrm{E}-01$ & $5.37 \mathrm{E}+01$ & $1.93 \mathrm{E}-06$ & $9.41 \mathrm{E}-08$ & $1.81 \mathrm{E}-04$ & $4.11 \mathrm{E}-11$ & $1.03 \mathrm{E}-11$ \\
\hline 241-AN-107 & 1.02E+00 & $5.29 \mathrm{E}-03$ & $9.98 \mathrm{E}-01$ & 4.07E+01 & $6.29 \mathrm{E}-02$ & $2.94 \mathrm{E}-04$ & $1.80 \mathrm{E}-01$ & $2.57 \mathrm{E}-05$ & $6.43 \mathrm{E}-06$ \\
\hline 24I-AP-101 & 8.39E-01 & 8.75E-03 & $2.31 \mathrm{E}+00$ & $4.45 \mathrm{E}+00$ & $3.11 \mathrm{E}-02$ & 1.17E-04 & $1.11 \mathrm{E}-01$ & $2.23 \mathrm{E}-06$ & $5.46 \mathrm{E}-07$ \\
\hline 241-AP-102 & 8.26E-01 & $1.22 \mathrm{E}-01$ & $5.38 \mathrm{E}-01$ & $3.28 \mathrm{E}+00$ & $1.01 \mathrm{E}-03$ & 8.58E-05 & $1.63 \mathrm{E}-01$ & & \\
\hline 241-AP-103 & $2.83 \mathrm{E}-02$ & $1.46 \mathrm{E}-03$ & $9.94 \mathrm{E}-02$ & $1.12 \mathbf{E}-01$ & $1.77 \mathrm{E}-06$ & $1.08 \mathrm{E}-06$ & 4.52E-03 & & \\
\hline 241-AP-104 & $4.54 \mathrm{E}-01$ & $1.59 \mathrm{E}-02$ & $8.42 \mathrm{E}-01$ & $1.50 \mathrm{E}+00$ & 4.07E-12 & $7.45 \mathrm{E}-14$ & $3.36 \mathrm{E}-09$ & 7.67E-13 & $4.88 \mathrm{E}-14$ \\
\hline 241-AP-105 & $2.28 \mathrm{E}+00$ & $6.94 \mathrm{E}-02$ & $5.26 \mathrm{E}-01$ & $4.79 \mathrm{E}+01$ & $1.50 \mathrm{E}-03$ & $3.50 \mathrm{E}-06$ & $1.39 \mathrm{E}-03$ & $1.05 \mathrm{E}-07$ & $2.61 \mathrm{E}-08$ \\
\hline 241-AP-106 & $1.97 \mathrm{E}+00$ & 5.05E-02 & $3.48 \mathrm{E}+00$ & $5.45 E+00$ & $2.87 \mathrm{E}-08$ & $3.64 \mathrm{E}-09$ & $6.86 \mathrm{E}-05$ & $3.51 \mathrm{E}-09$ & 8. $75 \mathrm{E}-10$ \\
\hline 241-AP-107 & $2.40 \mathrm{E}+00$ & $7.24 \mathrm{E}-02$ & $8.35 \mathrm{E}-01$ & $4.95 \mathrm{E}+01$ & $4.00 \mathrm{E}-02$ & $9.22 \mathrm{E}-05$ & $3.70 \mathrm{E}-02$ & $2.76 \mathrm{E}-06$ & $6.88 \mathrm{E}-07$ \\
\hline 241-AP-108 & $2.51 E-02$ & $2.21 \mathrm{E}-03$ & $9.15 \mathrm{E}-02$ & $4.99 \mathrm{E}-01$ & $5.33 \mathrm{E}-08$ & $2.02 \mathrm{E}-07$ & 1.67E-03 & & \\
\hline 241-AW-101 & $2.22 E+00$ & $2.22 \mathrm{E}-02$ & $5.07 \mathrm{E}+00$ & $2.46 \mathrm{E}+00$ & $7.13 \mathrm{E}-04$ & $1.51 \mathrm{E}-04$ & 3.27E-01 & $9.21 \mathrm{E}-07$ & 2.30E-07 \\
\hline \multicolumn{10}{|l|}{ 241-AW-102 } \\
\hline $241-\mathrm{AW}-103$ & $2.65 \mathrm{E}-02$ & $6.11 \mathrm{E}-04$ & $1.88 \mathrm{E}-01$ & $9.35 \mathrm{E}-01$ & $1.58 \mathrm{E}-07$ & $2.28 \mathrm{E}-05$ & $1.50 \mathrm{E}-02$ & & \\
\hline 241-AW-104 & $1.11 \mathrm{E}-02$ & $2.60 \mathrm{E}-03$ & $9.92 \mathrm{E}-02$ & $4.03 \mathrm{E}-01$ & $3.02 \mathrm{E}-09$ & $2.32 \mathrm{E}-09$ & 3.40E-06 & $1.97 \mathrm{E}-11$ & $3.87 \mathrm{E}-13$ \\
\hline 241-AW-105 & $1.40 \mathrm{E}-02$ & $2.41 \mathrm{E}-03$ & $4.41 \mathrm{E}-02$ & $2.39 \mathrm{E}-01$ & & 1.01E-07 & $7.86 \mathrm{E}-04$ & & \\
\hline \multicolumn{10}{|l|}{ 241-AW-106 } \\
\hline 241-AY-101 & $1.29 \mathrm{E}+00$ & $1.04 \mathrm{E}-02$ & $5.70 \mathrm{E}-01$ & $1.77 \mathrm{E}+00$ & $2.09 \mathrm{E}-03$ & $3.65 \mathrm{E}-04$ & $\begin{array}{c}1.08 \mathrm{E}+0 \\
0\end{array}$ & 1.33E-05 & $3.31 \mathrm{E}-06$ \\
\hline $241-A Y-102$ & 4.18E-05 & 2.77E-04 & 5.57E-01 & 3.93E-03 & $3.26 \mathrm{E}-06$ & 8.29E-08 & 1.13E-04 & $4.71 \mathrm{E}-08$ & $6.22 \mathrm{E}-12$ \\
\hline 241-AZ-101 & $2.17 \mathrm{E}-01$ & $2.08 \mathrm{E}-03$ & $1.76 \mathrm{E}-01$ & $1.38 \mathrm{E}-01$ & $1.09 \mathrm{E}-01$ & $6.54 \mathrm{E}-05$ & $2.73 \mathrm{E}-01$ & $7.12 \mathrm{E}-08$ & $1.78 \mathrm{E}-08$ \\
\hline 241-AZ-102 & $4.05 \mathrm{E}-02$ & $3.18 \mathrm{E}-05$ & $1.04 \mathrm{E}-01$ & $9.55 \mathrm{E}-02$ & $7.27 \mathrm{E}-05$ & $1.26 \mathrm{E}-05$ & $6.08 \mathrm{E}-02$ & $1.50 \mathrm{E}-06$ & $3.76 \mathrm{E}-07$ \\
\hline $241-S Y-101$ & $4.26 E+00$ & $8.35 \mathrm{E}-02$ & $2.42 \mathrm{E}+00$ & $1.37 \mathrm{E}+01$ & $2.52 \mathrm{E}-03$ & $1.11 \mathrm{E}-04$ & $4.26 \mathrm{E}-01$ & $1.17 \mathrm{E}-07$ & $2.92 \mathrm{E}-08$ \\
\hline $\begin{array}{l}241 \text {-SY- } \\
101 \text {-SOL }\end{array}$ & $6.73 \mathrm{E}-01$ & $5.73 \mathrm{E}-02$ & $5.79 E+00$ & $1.28 \mathrm{E}+01$ & 4.18E-03 & $3.49 \mathrm{E}-04$ & $1.32 \mathrm{E}-01$ & $1.19 \mathrm{E}-06$ & $3.24 \mathrm{E}-07$ \\
\hline 241-SY-102 & $1.46 \mathrm{E}-01$ & $1.11 \mathrm{E}-02$ & $3.61 \mathrm{E}-01$ & 9.31E-01 & $5.21 \mathrm{E}-12$ & & 1.12E-10 & 8. $34 \mathrm{E}-13$ & $1.14 \mathrm{E}-15$ \\
\hline 241-SY-103 & $2.23 \mathrm{E}+00$ & $5.12 \mathrm{E}-02$ & $7.67 \mathrm{E}-01$ & $3.18 \mathrm{E}+01$ & $3.47 \mathrm{E}-04$ & $3.00 \mathrm{E}-05$ & $4.65 \mathrm{E}-02$ & $9.01 \mathrm{E}-09$ & $2.25 \mathrm{E}-09$ \\
\hline $\begin{array}{c}\text { 241-SY-103- } \\
\text { SOL }\end{array}$ & $1.83 \mathrm{E}+00$ & $9.55 \mathrm{E}-02$ & $9.86 \mathrm{E}-01$ & $1.59 \mathrm{E}+00$ & $1.44 \mathrm{E}-02$ & $4.25 \mathrm{E}-04$ & $1.87 \mathrm{E}-01$ & $2.58 \mathrm{E}-06$ & $7.04 \mathrm{E}-07$ \\
\hline
\end{tabular}

241-AW-102 and 241-AW-106 are not included. They are the evaporator feed and receipt tanks, respectively and their projected compositions are too difficult to determine.

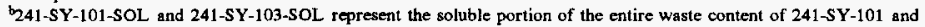
241-SY-103. 
WHC-SD-WM-TI-751

Revision 0

Table 2-1. Projected Double-Shell Tank Supernate Compositions for

Fiscal Year 2002. (4 Sheets)

\begin{tabular}{|c|c|c|c|c|c|c|c|c|c|}
\hline Supernatant & $\begin{array}{l}{ }^{241} \mathrm{Pu} \\
(\mathrm{Ci} / \mathrm{L})\end{array}$ & $\begin{array}{l}{ }^{241} \mathrm{Am} \\
(\mathrm{Ci} / \mathrm{L})\end{array}$ & $\begin{array}{c}\text { Volume, } \\
\text { (L) }\end{array}$ & $\begin{array}{c}\text { Quality of } \\
\text { Projec- } \\
\text { tion }\end{array}$ & & & & & \\
\hline 241-AN-101 & $3.36 \mathrm{E}-06$ & 1.57E-04 & $3.46 \mathrm{E}+06$ & $\mathbf{L}$ & & & & & \\
\hline 241-AN-102 & 4.86E-05 & $6.08 \mathrm{E}_{-} 05$ & $3.84 \mathrm{E}+06$ & $\mathbf{H}$ & & & & & \\
\hline 241-AN-103 & $1.28 \mathrm{E}-06$ & $2.30 \mathrm{E}-06$ & $3.62 E+06$ & $\mathrm{H}$ & & & $\because$ & & \\
\hline 241-AN-104 & $6.67 \mathrm{E}-06$ & 1.52E-06 & $3.02 \mathrm{E}+06$ & $\mathbf{H}$ & & & & $\because$ & \\
\hline 241-AN-105 & 6.67E-06 & $1.51 \mathrm{E}-06$ & $4.29 \mathrm{E}+06$ & $\mathbf{H}$ & & & & & \\
\hline 241-AN-106 & $3.29 \mathrm{E}-11$ & $8.19 \mathrm{E}-10$ & $4.00 \mathrm{E}+06$ & $\mathbf{L}$ & & & & & \\
\hline 241-AN-107 & $2.12 \mathrm{E}-05$ & $5.94 \mathrm{E}-04$ & $3.68 \mathrm{E}+06$ & $\mathbf{H}$ & & & & & \\
\hline 241-AP-101 & $1.72 \mathrm{E}-06$ & $6.98 \mathrm{E}-06$ & $4.31 \mathrm{E}+06$ & $\mathbf{M}$ & & & & & \\
\hline 241-AP-102 & & $4.19 \mathrm{E}-07$ & $4.16 \mathrm{E}+06$ & $\mathbf{H}$ & & & & & \\
\hline 241-AP-103 & & & $1.02 \mathbf{E}+05$ & $\mathbf{H}$ & & & & & \\
\hline 241-AP-104 & $1.21 \mathrm{E}-13$ & $1.13 \mathrm{E}-11$ & $4.22 \mathrm{E}+06$ & $\mathbf{M}$ & & & & & \\
\hline 241-AP-105 & $8.14 \mathrm{E}-08$ & $3.27 \mathrm{E}-07$ & $9.99 \mathrm{E}+04$ & $\mathbf{L}$ & & & & & \\
\hline 241-AP-106 & $2.73 \mathrm{E}-09$ & 1.01E-10 & $4.10 E+06$ & $\mathbf{L}$ & & & & . & \\
\hline 241-AP-107 & $2.19 \mathrm{E}-06$ & 8.62E-06 & $4.10 \mathrm{E}+06$ & $\mathbf{L}$ & & $\therefore \therefore$ & & & \\
\hline 241-AP-108 & & $9.35 \mathrm{E}-17$ & $1.02 \mathrm{E}+05$ & $\mathbf{H}$ & & & & 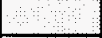 & 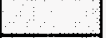 \\
\hline 241-AW-101 & $6.32 \mathrm{E}-07$ & $1.20 \mathrm{E}-06$ & $3.94 \mathrm{E}+06$ & $\mathbf{H}$ & & & & & - \\
\hline 241-AW-102 & & & & $N / A$ & & & & & \\
\hline 241-AW-103 & & & $5.41 \mathrm{E}+05$ & $\mathbf{H}$ & & $\therefore$ & & & \\
\hline 241-AW-104 & $1.30 \mathrm{E}-12$ & $1.43 \mathrm{E}-10$ & $2.27 \mathrm{E}+06$ & $\mathbf{M}$ & & & & $\therefore$ & \\
\hline 241-AW-105 & & & $1.00 \mathrm{E}+05$ & $\mathbf{H}$ & & & & & \\
\hline 241-AW-106 & & & & N/A & & & & & \\
\hline 241-AY-101 & $7.07 \mathrm{E}-06$ & $2.02 \mathrm{E}-05$ & $4.18 \mathrm{E}+06$ & $\mathbf{H}$ & & & & 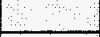 & \\
\hline 241-AY-102 & $2.11 \mathrm{E}-11$ & $9.37 \mathrm{E}-10$ & $2.90 \mathrm{E}+06$ & $\mathbf{M}$ & & & & $\therefore$ & 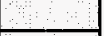 \\
\hline 241-AZ-101 & $6.11 \mathrm{E}-08$ & 4.47E-04 & $6.25 \mathrm{E}+05$ & $\mathbf{M}$ & & & & & \\
\hline 241-AZ-102 & $1.29 \mathrm{E}-06$ & $8.96 \mathrm{E}-08$ & $1.16 \mathrm{E}+06$ & $\mathbf{M}$ & & & & & \\
\hline 241-SY-101 & $8.34 \mathrm{E}-08$ & $7.30 \mathrm{E}-07$ & $6.81 \mathrm{E}+04$ & $\mathbf{H}$ & & & & & \\
\hline $\begin{array}{l}241-S Y- \\
101-S O L\end{array}$ & $7.94 \mathrm{E}-07$ & $1.09 \mathrm{E}-05$ & $7.37 \mathbf{E}+06$ & $\mathbf{L}$ & & & & & \\
\hline 241-SY-102 & $3.74 \mathrm{E}-15$ & $1.70 \mathrm{E}-11$ & $9.98 \mathrm{E}+04$ & $\mathbf{H}$ & & & & & \\
\hline 241-SY-103 & $4.88 \mathrm{E}-09$ & $3.22 \mathrm{E}-07$ & $6.43 E+05$ & $\mathbf{H}$ & & & & & \\
\hline $\begin{array}{l}241-S Y- \\
103-S O L\end{array}$ & $2.09 \mathrm{E}-06$ & $1.86 \mathrm{E}-05$ & $4.42 \mathrm{E}+06$ & L & & & & & \\
\hline
\end{tabular}

241-AW-102 and 241-AW-106 sre not included. They are the evaporator feed and receipt tanks, respectively and their projected compositions are too difficult to determine.

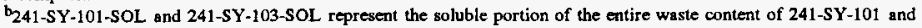
241-SY-103. 
WHC-SD-WM-TI-751

Revision 0

This page intentionally left blank. 
WHC-SD-WM-TI-751

Revision 0

\subsection{SAMPLE DATA}

Sample data for each DST, used as the starting point for the inventory projections, are shown in Table 3-1. Typically, the most recent sample data were used. Values for 241-SY-101-SOL and 241-SY-103-SOL were taken from Appendix A of the TWRS Process Flowsheet (Orme 1995). The ${ }^{240} \mathrm{Pu}$ activities were assumed to be $20 \mathrm{Ci}$ percent of the ${ }^{239 / 240} \mathrm{Pu}$ activities (ORNL 1995, Table 2.11). The ${ }^{99} \mathrm{Tc}$ values for DSTs 241-AN-101, 241-AN-104, 241-AN-105, 241-AP-101, 241-AP-107, 241-AW-104, 241-AY-101, 241-AY-102, and 241-SY-101 are arithmetic averages of ${ }^{99} \mathrm{Tc}$ values taken from DSTs with similar waste types. Less than values in laboratory reports were assumed to be zero and are not shown in the table. Components not listed in the laboratory reports were assumed to be zero and are not shown in the table. The decay dates for radionuclide concentrations are assumed to be the same as the sample date. The concentrations reported by the laboratory were not adjusted to achieve charge balances. 
WHC-SD-WM-TI-751

Revision 0

Table 3-1. Analytical Results for Double-Shell Tank Supernatants. ${ }^{a}$ (4 Sheets)

\begin{tabular}{|c|c|c|c|c|c|c|c|}
\hline Tank & $241-\mathrm{AN}-101$ & $241-\mathrm{AN}-102$ & 241-AN-103 & 241-AN-104 & 241-AN-105 & 241-AN-106 & $241-\mathrm{AN}-107$ \\
\hline Sample Date & $4 / 19 / 93$ & $10 / 21 / 94$ & $2 / 1 / 90$ & $10 / 10 / 93$ & $10 / 10 / 93$ & $10 / 1 / 93$ & $5 / 13 / 94$ \\
\hline $\mathrm{Al}(\mathrm{OH}) 4-(\mathrm{M})$ & $5.40 \mathrm{E}-01$ & $5.50 \mathrm{E}-01$ & $2.13 \mathrm{E}+00$ & $1.39 \mathrm{E}+00$ & $1.74 \mathrm{E}+00$ & $3.44 \mathrm{E}-01$ & $4.44 \mathrm{E}-02$ \\
\hline $\mathrm{Ba}+2(\mathrm{M})$ & $7.39 \mathrm{E}-07$ & & & & & $8.08 \mathrm{E}-05$ & \\
\hline $\mathrm{Ca}+2(\mathrm{M})$ & $1.59 \mathrm{E}-04$ & $1.10 \mathrm{E}-02$ & $2.16 \mathrm{E}-03$ & & & $2.25 \mathrm{E}-03$ & $1.37 \mathrm{E}-02$ \\
\hline $\mathrm{Cr}(\mathrm{OH}) 4-(\mathrm{M})$ & $3.62 \mathrm{E}-03$ & $5.50 \mathrm{E}-03$ & 1.63E-02 & $1.30 \mathrm{E}-02$ & $1.30 \mathrm{E}-02$ & $1.08 \mathrm{E}-02$ & 3.27E-03 \\
\hline $\mathrm{Fe}+3(\mathrm{M})$ & $4.94 \mathrm{E}-05$ & & $1.26 \mathrm{E}-03$ & 2.00E-04 & $2.00 \mathrm{E}-04$ & $1.24 \mathrm{E}-04$ & $2.69 \mathrm{E}-02$ \\
\hline $\mathrm{Hg}+2(\mathrm{M})$ & & & $7.98 \mathrm{E}-05$ & & & $2.49 \mathrm{E}-07$ & \\
\hline $\mathrm{K}+\mathrm{M})$ & 7.72E-02 & $1.00 \mathrm{E}-01$ & $3.89 \mathrm{E}-01$ & $1.83 \mathrm{E}-01$ & $1.57 \mathrm{E}-01$ & $2.76 \mathrm{E}-02$ & $5.42 \mathrm{E}-02$ \\
\hline \multicolumn{8}{|l|}{$\mathrm{La}+3(\mathrm{M})$} \\
\hline $\mathrm{Na}+(\mathrm{M})$ & $4.52 \mathrm{E}+00$ & $1.12 \mathrm{E}+01$ & $1.46 \mathrm{E}+01$ & $1.20 E+01$ & $1.20 \mathrm{E}+01$ & $3.93 \mathrm{E}+00$ & $8.65 E+00$ \\
\hline $\mathrm{Ni}+3(\mathrm{M})$ & $4.84 \mathrm{E}-05$ & $6.00 \mathrm{E}-03$ & $4.09 \mathrm{E}-04$ & & & & $7.67 \mathrm{E}-03$ \\
\hline $\mathrm{Pb}+4(\mathrm{M})$ & $7.05 \mathrm{E}-05$ & & $3.47 \mathrm{E}-04$ & & & $2.22 \mathrm{E}-03$ & $1.59 \mathrm{E}-03$ \\
\hline $\mathrm{U} \mathrm{g} / \mathrm{l}$ & $2.23 \mathrm{E}-02$ & & $5.18 \mathrm{E}-04$ & & & $1.68 \mathrm{E}-05$ & $2.25 E-01$ \\
\hline $\mathrm{CO} 3-2(\mathrm{M})$ & $2.17 \mathrm{E}-01$ & $1.12 \mathrm{E}+00$ & 1.49E-01 & $4.72 \mathrm{E}-01$ & $3.63 \mathrm{E}-01$ & $3.27 \mathrm{E}-01$ & $1.25 \mathrm{E}+00$ \\
\hline CL- (M) & $7.02 \mathrm{E}-02$ & $1.10 \mathrm{E}-01$ & $2.71 \mathrm{E}-01$ & $2.12 \mathrm{E}-01$ & $2.40 \mathrm{E}-01$ & $6.94 \mathrm{E}-02$ & $8.74 \mathrm{E}-02$ \\
\hline F- $(M)$ & & $1.10 \mathrm{E}-01$ & $3.87 \mathrm{E}-02$ & & & $3.24 \mathrm{E}-03$ & \\
\hline SO4-2 (M) & $2.85 \mathrm{E}-02$ & $1.50 \mathrm{E}-01$ & $1.67 \mathrm{E}-02$ & $6.82 \mathrm{E}-02$ & $6.82 \mathrm{E}-02$ & $2.24 \mathrm{E}-02$ & $1.43 \mathrm{E}-01$ \\
\hline No3- $(M)$ & $1.30 \mathrm{E}+00$ & $3.57 \mathrm{E}+00$ & $2.58 \mathrm{E}+00$ & $3.10 \mathrm{E}+00$ & $3.12 \mathrm{E}+00$ & $1.09 \mathrm{E}+00$ & $3.82 E+00$ \\
\hline NO2- $(\mathrm{M})$ & $8.78 \mathrm{E}-01$ & $1.82 \mathrm{E}+00$ & $3.00 \mathrm{E}+00$ & $1.92 \mathrm{E}+00$ & $2.61 \mathrm{E}+00$ & $6.44 \mathrm{E}-01$ & $1.07 \mathrm{E}+00$ \\
\hline PO4-3 (M) & $2.10 \mathrm{E}-02$ & $5.20 \mathrm{E}-02$ & $9.77 \mathrm{E}-03$ & $2.94 \mathrm{E}-02$ & $2.00 \mathrm{E}-02$ & $1.94 \mathrm{E}-01$ & $5.58 \mathrm{E}-03$ \\
\hline $\mathrm{OH}-(\mathrm{M})$ & $1.41 \mathrm{E}+00$ & $2.40 \mathrm{E}-01$ & $5,74 \mathrm{E}+00$ & $4.09 E+00$ & $3.64 \mathrm{E}+00$ & $4.74 \mathrm{E}-01$ & $2.00 \mathrm{E}-02$ \\
\hline $\operatorname{TOC}(\mathrm{g} / \mathrm{L})$ & $2.06 \mathrm{E}+00$ & $2.63 \mathrm{E}+01$ & $7.36 \mathrm{E}+00$ & $4.61 E+00$ & $3.88 \mathrm{E}+00$ & $3.26 \mathrm{E}+00$ & $4.29 E+01$ \\
\hline $90 \mathrm{Sr}(\mathrm{Ci} / \mathrm{L})$ & $8.90 \mathrm{E}-05$ & $7.89 \mathrm{E}-02$ & $1.30 \mathrm{E}-02$ & $9.84 \mathrm{E}-03$ & $3.23 E-03$ & $2.00 \mathrm{E}-03$ & $9.23 \mathrm{E}-02$ \\
\hline $99 \mathrm{Tc}(\mathrm{Ci} / \mathrm{L})^{(\mathrm{b})}$ & $2.66 \mathrm{E}-05$ & $3.10 \mathrm{E}-04$ & $1.70 \mathrm{E}-04$ & $1.31 \mathrm{E}-04$ & $1.31 \mathrm{E}-04$ & $6.92 E-05$ & $3.10 \mathrm{E}-04$ \\
\hline $137 \mathrm{Cs}(\mathrm{Ci} / \mathrm{L})$ & $1.22 \mathrm{E}-01$ & $2.58 \mathrm{E}-01$ & $7.51 \mathrm{E}-01$ & $7.56 \mathrm{E}-01$ & $5.04 \mathrm{E}-01$ & $1.85 \mathrm{E}-01$ & $2.60 \mathrm{E}-01$ \\
\hline $154 \mathrm{Eu}(\mathrm{Ci} / \mathrm{L})$ & & $7.96 \mathrm{E}-04$ & & & & & \\
\hline $238 \mathrm{U}(\mathrm{Ci} / \mathrm{L})$ & $7.49 \mathrm{E}-09$ & & $1.74 \mathrm{E}-10$ & & & $5.64 \mathrm{E}-12$ & $7.55 \mathrm{E}-08$ \\
\hline $239 \mathrm{P} \sim(\mathrm{Ci} / \mathrm{L})$ & $2.48 \mathrm{E}-08$ & $5.89 \mathrm{E}-05$ & $1.90 \mathrm{E}-06$ & $8.32 \mathrm{E}-06$ & $8.32 \mathrm{E}-06$ & $3.02 \mathrm{E}-08$ & $2.71 \mathrm{E}-05$ \\
\hline $240 \mathrm{Pu}(\mathrm{Cl} / \mathrm{L})$ & $6.20 \mathrm{E}-09$ & $1.47 \mathrm{E}-05$ & 4.75E-07 & $2.08 \mathrm{E}-06$ & $2.08 \mathrm{E}-06$ & $7.55 \mathrm{E}-09$ & $6.78 \mathrm{E}-06$ \\
\hline $241 \mathrm{Pu}(\mathrm{Ci} / \mathrm{L})$ & $3.90 \mathrm{E}-08$ & $9.28 \mathrm{E}-05$ & $2.99 \mathrm{E}-06$ & $1.31 \mathrm{E}-05$ & $1.31 \mathrm{E}-05$ & $4.76 \mathrm{E}-08$ & 4.27E-05 \\
\hline $241 \mathrm{Am}(\mathrm{Ci} / \mathrm{L})$ & $5.43 \mathrm{E}-07$ & $6.20 \mathrm{E}-05$ & $2.30 \mathrm{E}-06$ & $1.52 \mathrm{E}-06$ & $1.51 \mathrm{E}-06$ & $6.02 \mathrm{E}-07$ & $6.26 \mathrm{E}-04$ \\
\hline Volume, (L) & $2.72 \mathrm{E}+06$ & $3.79 \mathrm{E}+06$ & $3.61 \mathrm{E}+06$ & $4.00 E+06$ & $4.28 \mathrm{E}+06$ & $7.95 \mathrm{E}+04$ & $3.52 \mathrm{E}+06$ \\
\hline
\end{tabular}


WHC-SD-WM-TI-751

Revision 0

Table 3-1. Analytical Results for Double-Shell Tank Supernatants. ${ }^{8}$ (4 Sheets)

\begin{tabular}{|c|c|c|c|c|c|c|c|}
\hline Tank & 241-AP-101 & 241-AP-102 & 241-AP-103 & 241-AP-104 & 241-AP-105 & 241-AP-106 & 241-AP-107 \\
\hline Sample Date & $7 / 20 / 93$ & $4 / 30 / 93$ & $11 / 25 / 91$ & $6 / 1 / 88$ & $3 / 14 / 93$ & $3 / 17 / 93$ & $8 / 1 / 93$ \\
\hline $\mathrm{Al}(\mathrm{OH}) 4-(\mathrm{M})$ & $2.47 \mathrm{E}-05$ & $4.30 \mathrm{E}-01$ & 9.11E-03 & $3.60 \mathrm{E}-05$ & $4.33 \mathrm{E}-01$ & $7.81 \mathrm{E}-03$ & $3.93 \mathrm{E}-05$ \\
\hline $\mathrm{Ba}+2(\mathrm{M})$ & 1.65E-06 & $2.07 \mathrm{E}-06$ & & $3.53 \mathrm{E}-06$ & $4.65 \mathrm{E}-06$ & $1.17 \mathrm{E}-06$ & $1.81 \mathrm{E}-07$ \\
\hline $\mathrm{Ca}+2(\mathrm{M})$ & $5.02 \mathrm{E}-04$ & & & $2.18 \mathrm{E}-05$ & $1.66 \mathrm{E}-03$ & & 5.02E-04 \\
\hline $\mathrm{Cr}(\mathrm{OH}) 4-(\mathrm{M})$ & $7.56 \mathrm{E}-07$ & $1.19 \mathrm{E}-02$ & $8.44 \mathrm{E}-05$ & $3.17 \mathrm{E}-05$ & $3.60 \mathrm{E}-03$ & $9.12 \mathrm{E}-05$ & \\
\hline $\mathrm{Fe}+3(\mathrm{M})$ & $5.03 E-06$ & $6.82 E-05$ & $2.20 \mathrm{E}-05$ & 3.99E-05 & $1.18 \mathrm{E}-04$ & $1.23 \mathrm{E}-04$ & \\
\hline $\mathrm{Hg}+2(\mathrm{M})$ & & & $2.49 \mathrm{E}-08$ & & & & \\
\hline $\mathrm{K}+(\mathrm{M})$ & & $3.30 \mathrm{E}-02$ & $3.89 \mathrm{E}-02$ & $2.48 \mathrm{E}-04$ & $7.93 \mathrm{E}-01$ & $2.09 \mathrm{E}-02$ & \\
\hline \multicolumn{8}{|l|}{$\mathrm{La}+3(\mathrm{M})$} \\
\hline $\mathrm{Na}+(\mathrm{M})$ & $1.94 \mathrm{E}-01$ & $4.43 \mathrm{E}+00$ & $2.31 \mathrm{E}-01$ & $4.91 E-01$ & $7.26 \mathrm{E}+00$ & $2.40 \mathrm{E}-01$ & $9.91 \mathrm{E}-02$ \\
\hline $\mathrm{Ni}+3(\mathrm{M})$ & & $4.53 \mathrm{E}-04$ & & $3.31 \mathrm{E}-06$ & $1.86 \mathrm{E}-04$ & $6.95 \mathrm{E}-06$ & \\
\hline $\mathrm{Pb}+4(\mathrm{M})$ & $2.15 \mathrm{E}-06$ & $1.59 \mathrm{E}-05$ & & $1,87 \mathrm{E}-05$ & $2.60 \mathrm{E}-05$ & & \\
\hline $\mathrm{U}(\mathrm{g} / \mathrm{l})$ & $2.12 \mathrm{E}-04$ & $3.52 \mathrm{E}-03$ & $3.69 \mathrm{E}-03$ & $2.20 \mathrm{E}-04$ & $4.22 \mathrm{E}-02$ & $3.71 \mathrm{E}-03$ & $2.34 \mathrm{E}-05$ \\
\hline $\mathrm{CO3-2}(\mathrm{M})$ & $1.58 \mathrm{E}-02$ & $4.47 \mathrm{E}-01$ & $4.28 \mathrm{E}-02$ & & $3.75 \mathrm{E}-01$ & $4.30 \mathrm{E}-02$ & $2.47 \mathrm{E}-02$ \\
\hline$\overline{C L-(M)}$ & & $8.18 \mathrm{E}-02$ & $1.40 \mathrm{E}-03$ & $1.03 \mathrm{E}-03$ & $6.66 \mathrm{E}-02$ & $1.59 \mathrm{E}-03$ & \\
\hline F- (M) & $3.61 \mathrm{E}-03$ & & $6.79 \mathrm{E}-03$ & $8.74 \mathrm{E}-04$ & $8.00 \mathrm{E}-02$ & $9.11 \mathrm{E}-03$ & $7.47 \mathrm{E}-03$ \\
\hline SO4-2 (M) & $8.97 \mathrm{E}-04$ & $4.70 \mathrm{E}-02$ & $3.64 \mathrm{E}-03$ & $1.64 \mathrm{E}-02$ & $2.52 \mathrm{E}-02$ & $1.46 \mathrm{E}-03$ & $2.11 \mathrm{E}-03$ \\
\hline NO3- (M) & $2.55 \mathrm{E}-02$ & $1.26 \mathrm{E}+00$ & $6.52 \mathrm{E}-02$ & $3.10 \mathrm{E}-05$ & $2.66 \mathrm{E}+00$ & $6.82 \mathrm{E}-02$ & $1.65 \mathrm{E}-02$ \\
\hline NO2- (M) & $2.41 \mathrm{E}-02$ & $8.26 \mathrm{E}-01$ & $2.83 \mathrm{E}-02$ & & $1.05 \mathrm{E}+00$ & $2.52 \mathrm{E}-02$ & $2.41 \mathrm{E}-02$ \\
\hline PO4-3 (M) & & $1.22 \mathrm{E}-01$ & $1.46 \mathrm{E}-03$ & $1.71 \mathrm{E}-01$ & $4.63 \mathrm{E}-03$ & $2.22 \mathrm{E}-03$ & \\
\hline $\mathrm{OH}-(\mathrm{M})$ & $1.07 \mathrm{E}-01$ & $5.38 \mathrm{E}-01$ & $9.94 \mathrm{E}-02$ & & $3.18 \mathrm{E}+00$ & $8.41 \mathrm{E}-02$ & $1.56 \mathrm{E}-02$ \\
\hline TOC $(g / L)$ & $2.37 \mathrm{E}-02$ & $3.28 \mathrm{E}+00$ & $1.12 \mathrm{E}-01$ & $1.90 \mathrm{E}-01$ & $2.75 \mathrm{E}+00$ & $4.86 \mathrm{E}-01$ & $5.20 \mathrm{E}-02$ \\
\hline $90 \mathrm{Sr}(\mathrm{Ci} / \mathrm{L})$ & $5.63 \mathrm{E}-07$ & $1.44 \mathrm{E}-03$ & $2.62 \mathrm{E}-06$ & $3.27 \mathrm{E}-08$ & $2.08 \mathrm{E}-04$ & $6.89 \mathrm{E}-07$ & $1.18 \mathrm{E}-08$ \\
\hline $99 \mathrm{Tc}(\mathrm{Ci} / \mathrm{L})$ & $2.66 \mathrm{E}-05$ & $8.58 \mathrm{E}-05$ & $1.08 \mathrm{E}-06$ & $4.81 \mathrm{E}-08$ & $6.99 \mathrm{E}-05$ & $1.34 \mathrm{E}-05$ & $2.66 \mathrm{E}-05$ \\
\hline $137 \mathrm{Cs}(\mathrm{Ci} / \mathrm{L})$ & $3.26 \mathrm{E}-05$ & $2.28 \mathrm{E}-01$ & $6.54 \mathrm{E}-03$ & $5.81 \mathrm{E}-07$ & $2.27 \mathrm{E}-01$ & $4.57 \mathrm{E}-03$ & $1.23 \mathrm{E}-07$ \\
\hline $154 \mathrm{Eu}(\mathrm{Ci} / \mathrm{L})$ & $5.86 \mathrm{E}-07$ & & & & & & \\
\hline $238 \mathrm{U}(\mathrm{Ci} / \mathrm{L})$ & $7.12 \mathrm{E}-11$ & $1.18 \mathrm{E}-09$ & $1.24 \mathrm{E}-09$ & $7.39 \mathrm{E}-11$ & $1.42 \mathrm{E}-08$ & $1.25 \mathrm{E}-09$ & $7.86 \mathrm{E}-12$ \\
\hline $239 \mathrm{Pu}(\mathrm{Ci} / \mathrm{L})$ & & & & 2.55E-09 & $1.26 \mathrm{E}-07$ & & \\
\hline $240 \mathrm{Pu}(\mathrm{Ci} / \mathrm{L})$ & & & & $6.38 \mathrm{E}-10$ & $3.15 \mathrm{E}-08$ & & \\
\hline $241 \mathrm{Pu}(\mathrm{Ci} / \mathrm{L})$ & & & & 4.02E-09 & $1.98 \mathrm{E}-07$ & & \\
\hline $241 \mathrm{Am}(\mathrm{Ci} / \mathrm{L})$ & & $4.19 \mathrm{E}-07$ & & $7.84 \mathrm{E}-10$ & $4.06 \mathrm{E}-07$ & $9.54 \mathrm{E}-11$ & \\
\hline Volume, (L) & $4.01 E+06$ & $4.17 \mathrm{E}+06$ & $4.28 \mathrm{E}+06$ & $6.81 \mathrm{E}+04$ & $3.10 \mathrm{E}+06$ & $4.27 \mathrm{E}+06$ & $4.20 \mathrm{E}+06$ \\
\hline
\end{tabular}


WHC-SD-WM-TI-751

Revision 0

Table 3-1. Analytical Results for Double-Shell Tank Supernatants. ${ }^{a}$ (4 Sheets)

\begin{tabular}{|c|c|c|c|c|c|c|c|}
\hline Tank & 241-AP-108 & 241-AW-101 & 241-AW-102 & 241-AW-103 & 241-AW-104 & 241-AW-105 & $241-A W-106$ \\
\hline Sample Date & $3 / 19 / 94$ & $6 / 28 / 90$ & $11 / 1 / 93$ & 9/29/94 & $9 / 27 / 94$ & $7 / 18 / 91$ & $6 / 1 / 91$ \\
\hline $\mathrm{Al}(\mathrm{OH}) 4-(\mathrm{M})$ & $1.54 \mathrm{E}-03$ & $1.03 E+00$ & $1.81 \mathrm{E}-02$ & $1.54 \mathrm{E}-03$ & $3.67 \mathrm{E}-03$ & $2.84 \mathrm{E}-02$ & $6.21 \mathrm{E}-02$ \\
\hline $\mathrm{Ba}+2(\mathrm{M})$ & & & $9.47 \mathrm{E}-06$ & $4.08 \mathrm{E}-06$ & & $9.92 \mathrm{E}-07$ & $1.26 \mathrm{E}-05$ \\
\hline $\mathrm{Ca}+2(\mathrm{M})$ & $4.27 \mathrm{E}-05$ & $8.26 \mathrm{E}-04$ & & $1.54 \mathrm{E}-04$ & & $1.18 \mathrm{E}-03$ & \\
\hline $\mathrm{Cr}(\mathrm{OH}) 4-(\mathrm{M})$ & $3.90 \mathrm{E}-05$ & $3.10 \mathrm{E}-03$ & $2.05 \mathrm{E}-04$ & $1.40 \mathrm{E}-03$ & & $1.01 \mathrm{E}-03$ & $4.41 \mathrm{E}-04$ \\
\hline $\mathrm{Fe}+3(\mathrm{M})$ & 9.13E-06 & & $5.65 \mathrm{E}-04$ & & & $3.17 \mathrm{E}-05$ & $1.34 \mathrm{E}-03$ \\
\hline $\mathrm{Hg}+2(\mathrm{M})$ & & & 2.49E-08 & & & & $5.21 \mathrm{E}-08$ \\
\hline $\mathrm{K}+(\mathrm{M})$ & & $1.07 \mathrm{E}+00$ & & $5.22 \mathrm{E}-01$ & & $3.76 \mathrm{E}-01$ & \\
\hline \multicolumn{8}{|l|}{$\mathrm{La}+3(\mathrm{M})$} \\
\hline $\mathrm{Na}+(\mathrm{M})$ & $1.20 \mathrm{E}-01$ & $1.00 \mathrm{E}+01$ & $7.30 \mathrm{E}-01$ & $8.52 \mathrm{E}-01$ & $3.60 \mathrm{E}-01$ & $1.19 \mathrm{E}+00$ & $1.73 \mathrm{E}+00$ \\
\hline $\mathrm{Ni}+3(\mathrm{M})$ & & & & $5.05 \mathrm{E}-05$ & & & \\
\hline $\mathrm{Pb}+4(\mathrm{M})$ & & $1.46 \mathrm{E}-03$ & $3.86 \mathrm{E}-05$ & & & & \\
\hline $\mathrm{U}(\mathrm{g} / \mathrm{l})$ & $1.35 \mathrm{E}-02$ & $2.24 \mathrm{E}-01$ & $1.54 \mathrm{E}-08$ & $9.87 \mathrm{E}-03$ & & $8.07 \mathrm{E}-03$ & $8.99 \mathrm{E}-03$ \\
\hline $\operatorname{co3}-2(M)$ & $8.10 \mathrm{E}-03$ & & & $7.68 \mathrm{E}-02$ & $3.48 \mathrm{E}-02$ & $5.11 \mathrm{E}-02$ & \\
\hline CL- (M) & $1.59 \mathrm{E}-03$ & $1.46 \mathrm{E}-01$ & $1.31 \mathrm{E}-02$ & $3.81 \mathrm{E}-03$ & $1.67 \mathrm{E}-03$ & $5.19 \mathrm{E}-03$ & $1.51 \mathrm{E}-02$ \\
\hline F- (M) & $1.79 \mathrm{E}-03$ & & $2.12 \mathrm{E}-01$ & $9.16 \mathrm{E}-01$ & $6.47 \mathrm{E}-04$ & $7.11 \mathrm{E}-01$ & $3.43 \mathrm{E}-01$ \\
\hline SO4-2 (M) & $2.09 \mathrm{E}-03$ & $1.07 \mathrm{E}-02$ & $2.43 \mathrm{E}-02$ & $5.86 \mathrm{E}-04$ & $3.15 \mathrm{E}-03$ & $7.94 \mathrm{E}-03$ & $3.56 \mathrm{E}-02$ \\
\hline NO3-(M) & $2.52 \mathrm{E}-02$ & $3.45 \mathrm{E}+00$ & $2.31 \mathrm{E}-01$ & $5.66 \mathrm{E}-02$ & $1.74 \mathrm{E}-01$ & $5.13 \mathrm{E}-02$ & $5.88 \mathrm{E}-01$ \\
\hline NO2- (M) & $2.20 \mathrm{E}-02$ & $2.22 \mathrm{E}+00$ & $5.59 \mathrm{E}-02$ & $2.65 \mathrm{E}-02$ & $4.72 \mathrm{E}-02$ & $2.11 \mathrm{E}-02$ & $2.08 \mathrm{E}-01$ \\
\hline $\mathrm{PO} 4-3(\mathrm{M})$ & $9.76 \mathrm{E}-04$ & $2.22 \mathrm{E}-02$ & $1.84 \mathrm{E}-03$ & $6.11 \mathrm{E}-04$ & & $1.43 \mathrm{E}-03$ & $7.67 \mathrm{E}-03$ \\
\hline $\mathrm{OH}-(\mathrm{M})$ & $7.35 \mathrm{E}-03$ & $5.07 \mathrm{E}+00$ & $1.70 \mathrm{E}-01$ & $1.88 \mathrm{E}-01$ & $1.27 \mathrm{E}-05$ & 6.33E-01 & $5.88 \mathrm{E}-01$ \\
\hline TOC $(\mathrm{g} / \mathrm{L})$ & & $2.46 \mathrm{E}+00$ & $4.91 \mathrm{E}-01$ & $9.35 \mathrm{E}-01$ & $4.60 \mathrm{E}-01$ & $1.57 \mathrm{E}+00$ & $1.05 E+00$ \\
\hline $90 \mathrm{Sr}(\mathrm{Ci} / \mathrm{L})$ & $7.72 \mathrm{E}-05$ & $1.09 \mathrm{E}-03$ & $4.53 \mathrm{E}-05$ & $2.20 \mathrm{E}-07$ & $7.79 \mathrm{E}-07$ & & $2.95 \mathrm{E}-05$ \\
\hline $99 \mathrm{Tc}(\mathrm{Ci} / \mathrm{L})$ & $3.08 \mathrm{E}-07$ & $1.51 \mathrm{E}-04$ & & $2.28 \mathrm{E}-05$ & $2.66 \mathrm{E}-05$ & $1.44 \mathrm{E}-05$ & $1.68 \mathrm{E}-05$ \\
\hline $137 \mathrm{Cs}(\mathrm{Ci} / \mathrm{L})$ & $4.29 \mathrm{E}-03$ & $4.89 \mathrm{E}-01$ & $1.52 \mathrm{E}-02$ & $2.06 \mathrm{E}-02$ & $4.54 \mathrm{E}-03$ & $1.64 \mathrm{E}-01$ & $5.07 \mathrm{E}-02$ \\
\hline $154 \mathrm{Eu}(\mathrm{Ci} / \mathrm{L})$ & & & $2.17 \mathrm{E}-05$ & & & & \\
\hline $238 \mathrm{U}(\mathrm{Ci} / \mathrm{L})$ & $4.54 \mathrm{E}-09$ & $7.53 \mathrm{E}-08$ & $5.17 \mathrm{E}-15$ & $3.32 \mathrm{E}-09$ & & $2.71 \mathrm{E}-09$ & $3.02 \mathrm{E}-09$ \\
\hline $239 \mathrm{Pu}(\mathrm{Ci} / \mathrm{L})$ & & $9.21 \mathrm{E}-07$ & $3.61 E-07$ & & $1.30 \mathrm{E}-08$ & & \\
\hline $240 \mathrm{Pu}(\mathrm{Ci} / \mathrm{L})$ & & $2.30 \mathrm{E}-07$ & $9.03 \mathrm{E}-08$ & & $3.25 \mathrm{E}-09$ & & \\
\hline $24 \mathrm{IPu}(\mathrm{Ci} / \mathrm{L})$ & & $1.45 \mathrm{E}-06$ & $5.69 \mathrm{E}-07$ & & $2.05 \mathrm{E}-08$ & & \\
\hline $241 \mathrm{Am}(\mathrm{Ci} / \mathrm{L})$ & & $1.20 \mathrm{E}-06$ & $2.57 \mathrm{E}-07$ & & $2.98 \mathrm{E}-07$ & & \\
\hline Volume, (L) & $4.28 \mathrm{E}+06$ & $4.07 \mathrm{E}+06$ & $3.71 \mathrm{E}+06$ & $1.21 \mathrm{E}+06$ & $4.25 \mathrm{E}+06$ & $2.94 \mathrm{E}+06$ & $4.20 \mathrm{E}+06$ \\
\hline
\end{tabular}


WHC-SD-WM-TI-751

Revision 0

Table 3-1. Analytical Results for Double-Shell Tank Supernatants. ${ }^{a}$ (4 Sheets)

\begin{tabular}{|c|c|c|c|c|c|c|c|}
\hline Tank & $241-A Y-101$ & 241-AY-102 & 241-AZ-101 & 241-AZ-102 & 241-SY-101 & 241-SY-102 & 241-SY-103 \\
\hline Sample Date & $2 / 28 / 85$ & $12 / 1 / 94$ & $3 / 3 / 95$ & $2 / 23 / 95$ & $5 / 1 / 91$ & $3 / 10 / 94$ & $9 / 1 / 94$ \\
\hline $\mathrm{Al}(\mathrm{OH}) 4-(\mathrm{M})$ & $6.59 \mathrm{E}-02$ & $5.08 \mathrm{E}-04$ & 3.45E-01 & $5.74 \mathrm{E}-03$ & $1.58 \mathrm{E}+00$ & 2.35E-02 & $1.54 \mathrm{E}+00$ \\
\hline $\mathrm{Ba}+2(\mathrm{M})$ & $4.37 E-05$ & & & & & & \\
\hline $\mathrm{Ca}+2(\mathrm{M})$ & $5.41 \mathrm{E}-03$ & & & & $1.87 \mathrm{E}-02$ & 7.22E-05 & $2.95 \mathrm{E}-03$ \\
\hline $\mathrm{Cr}(\mathrm{OH}) 4-(\mathrm{M})$ & $6.25 \mathrm{E}-04$ & . & $1.23 \mathrm{E}-02$ & $1.86 \mathrm{E}-02$ & $1.56 \mathrm{E}-03$ & $1.85 \mathrm{E}-04$ & $6.33 \mathrm{E}-04$ \\
\hline $\mathrm{Fe}+3(\mathrm{M})$ & $1.86 \mathrm{E}-04$ & & & & $3.51 \mathrm{E}-04$ & & \\
\hline \multicolumn{8}{|l|}{$\mathrm{Hg}+2(\mathrm{M})$} \\
\hline $\mathrm{K}+(\mathrm{M})$ & $8.54 \mathrm{E}-03$ & & $1.03 \mathrm{E}-01$ & & $1.27 \mathrm{E}-01$ & $6.19 \mathrm{E}-02$ & $1.00 \mathrm{E}-01$ \\
\hline $\mathrm{La}+3(\mathrm{M})$ & $4.61 \mathrm{E}-04$ & & & & & & \\
\hline $\mathrm{Na}+(\mathrm{M})$ & $2.47 \mathrm{E}+00$ & $1.07 \mathrm{E}-01$ & $4.14 \mathrm{E}+00$ & $2.31 \mathrm{E}+00$ & $1.17 \mathrm{E}+01$ & $7.52 \mathrm{E}-01$ & $9.20 \mathrm{E}+00$ \\
\hline $\mathrm{Ni}+3(\mathrm{M})$ & $1.11 \mathrm{E}-03$ & & & & $8.86 \mathrm{E}-04$ & & $8.27 \mathrm{E}-04$ \\
\hline \multicolumn{8}{|l|}{$\mathrm{Pb}+4(\mathrm{M})$} \\
\hline $\mathrm{U}(\mathrm{g} / \mathrm{l})$ & $1.97 \mathrm{E}-02$ & & & $1.54 \mathrm{E}+00$ & $3.39 \mathrm{E}-03$ & & $2.82 \mathrm{E}-03$ \\
\hline $\mathrm{CO} 3-2(\mathrm{M})$ & $2.83 \mathrm{E}-01$ & $4.00 \mathrm{E}-02$ & $4.82 \mathrm{E}-01$ & $4.80 \mathrm{E}-01$ & & $8.58 \mathrm{E}-07$ & \\
\hline $\mathrm{CL}-(\mathrm{M})$ & $1.30 \mathrm{E}-02$ & $2.66 \mathrm{E}-03$ & $4.91 \mathrm{E}-03$ & & 3.89E-01 & $5.64 \mathrm{E}-03$ & $3.27 \mathrm{E}-01$ \\
\hline$F-(M)$ & $2.94 \mathrm{E}-02$ & $6.26 \mathrm{E}-04$ & 8.32E-02 & $5.29 \mathrm{E}-02$ & & $3.96 \mathrm{E}-02$ & \\
\hline SO4-2 (M) & $6.54 \mathrm{E}-02$ & 1.67E-03 & $1.60 \mathrm{E}-01$ & $1.80 \mathrm{E}-01$ & $1.43 \mathrm{E}-02$ & $5.43 \mathrm{E}-03$ & \\
\hline NO3- $(\mathrm{M})$ & $8.19 E-01$ & $8.48 \mathrm{E}-03$ & $1.06 \mathrm{E}+00$ & $3.84 \mathrm{E}-01$ & $3.68 \mathrm{E}+00$ & $3.79 \mathrm{E}-01$ & $2.89 E+00$ \\
\hline NO2- $(\mathrm{M})$ & $2.54 \mathrm{E}-01$ & $1.92 \mathrm{E}-02$ & $1.23 \mathrm{E}+00$ & $6.00 \mathrm{E}-01$ & $4.26 \mathrm{E}+00$ & $7.01 \mathrm{E}-02$ & $3.11 \mathrm{E}+00$ \\
\hline PO4-3 (M) & $1.03 \mathrm{E}-02$ & $6.91 \mathrm{E}-04$ & $1.38 \mathrm{E}-02$ & & $8.35 \mathrm{E}-02$ & $4.18 \mathrm{E}-03$ & $3.28 \mathrm{E}-02$ \\
\hline $\mathrm{OH}-(\mathrm{M})$ & $4.47 \mathrm{E}-01$ & $1.81 \mathrm{E}-02$ & $6.70 \mathrm{E}-01$ & $1.11 \mathrm{E}-01$ & $2.42 \mathrm{E}+00$ & $2.04 \mathrm{E}-01$ & $1.68 E+00$ \\
\hline TOC $(\mathrm{g} / \mathrm{L})$ & $5.74 \mathrm{E}+00$ & $8.00 \mathrm{E}-02$ & $1.03 E+00$ & $1.50 \mathrm{E}+00$ & $1.37 \mathrm{E}+01$ & $2.50 \mathrm{E}-01$ & $9.64 E+00$ \\
\hline $90 \mathrm{Sr}(\mathrm{Ci} / \mathrm{L})$ & $2.00 \mathrm{E}-01$ & $2.00 \mathrm{E}-04$ & $1.20 \mathrm{E}-03$ & $1.76 \mathrm{E}-03$ & $3.78 \mathrm{E}-03$ & $1.09 \mathrm{E}-06$ & $2.21 \mathrm{E}-03$ \\
\hline $99 \mathrm{Tc}(\mathrm{Ci} / \mathrm{L})$ & $3.10 \mathrm{E}-04$ & $2.66 \mathrm{E}-05$ & $3.56 \mathrm{E}-04$ & $1.59 \mathrm{E}-04$ & $1.11 \mathrm{E}-04$ & & $1.11 \mathrm{E}-04$ \\
\hline $137 \mathrm{Cs}(\mathrm{Ci} / \mathrm{L})$ & $1.40 \mathrm{E}-01$ & $3.40 \mathrm{E}-03$ & $1.59 \mathrm{E}+00$ & $1.04 \mathrm{E}+00$ & $6.24 \mathrm{E}-01$ & $6.57 \mathrm{E}-03$ & $2.88 \mathrm{E}-01$ \\
\hline 154Eu $(\mathrm{Ci} / \mathrm{L})$ & & & $3.58 \mathrm{E}-03$ & $3.62 \mathrm{E}-04$ & & & \\
\hline $238 \mathrm{U}(\mathrm{Ci} / \mathrm{L})$ & $6.62 \mathrm{E}-09$ & & & $5.17 E-07$ & $1.14 \mathrm{E}-09$ & & $9.48 \mathrm{E}-10$ \\
\hline $239 \mathrm{Pu}(\mathrm{Ci} / \mathrm{L})$ & $9.68 \mathrm{E}-06$ & $2.08 \mathrm{E}-08$ & $5.31 \mathrm{E}-07$ & 2.08E-05 & $1.17 \mathrm{E}-07$ & 2.47E-07 & $3.33 E-08$ \\
\hline $240 \mathrm{Pu}(\mathrm{Ci} / \mathrm{L})$ & $2.42 E-06$ & 5.20E-09 & $1.33 \mathrm{E}-07$ & $5.20 \mathrm{E}-06$ & 2.92E-08 & $6.18 \mathrm{E}-08$ & 8.33E-09 \\
\hline $241 \mathrm{Pu}(\mathrm{Ci} / \mathrm{L})$ & $1.52 \mathrm{E}-05$ & $3.28 \mathrm{E}-08$ & $8.36 \mathrm{E}-07$ & $3.28 \mathrm{E}-05$ & $1.84 E-07$ & $3.89 \mathrm{E}-07$ & $5.24 \mathrm{E}-08$ \\
\hline $241 \mathrm{Am}(\mathrm{Ci} / \mathrm{L})$ & $3.03 \mathrm{E}-05$ & $6.90 \mathrm{E}-07$ & $2.58 \mathrm{E}-05$ & 1.24E-06 & $7.30 \mathrm{E}-07$ & & $1.19 \mathrm{E}-06$ \\
\hline Volume, (L) & $3.04 E+06$ & $2.55 \mathrm{E}+06$ & $3.43 E+06$ & $3.32 \mathrm{E}+06$ & $1.15 \mathrm{E}+06$ & $2.56 \mathrm{E}+06$ & $2.49 \mathrm{E}+06$ \\
\hline
\end{tabular}

${ }^{\mathrm{s}}$ Less than values were assumed to be zero.

${ }^{b}$ The ${ }^{99} \mathrm{Tc}$ values for 241-AN-101, 241-AN-104, 241-AN-105, 241-AP-101, 241-AP-107, 241-AW-104, 241-AY-101, 241-AY-102, and 241-SY-101 are arithmetic averages of wastes with similar waste types. 
WHC-SD-WM-TI-751

Revision 0

This page intentionally left blank. 


\subsection{PROJECTING DOUBLE-SHELL TANK SUPERNATANT COMPOSITIONS}

Projecting the DST supernatant compositions is done in two steps. First, historical transaction records are used to project compositions to the present in Section 5.1. Then, operational waste volume projections are used to estimate future compositions in Section 5.2.

\subsection{USING HISTORICAL TRANSACTION RECORDS TO PROJECT COMPOSITIONS TO THE PRESENT}

The dates the samples were taken range from February 28, 1985, to March 3, 1995. For older samples, historical transaction records (Tank Farm Process Engineering Group) that chronicle transfers of waste into and out of all the DSTs can be used to project compositions from the sample date to the near present. The historical transaction records used in this report include records through February 1996. An example of part of a historical transaction sheet for 241-AW-105 is shown in Table 4-1. Tank 241-AW-105 has been a receiver for plutonium-uranium extraction miscellaneous wastes (PXMSC) since 1983. The last sampling event for 241-AW-105 was in May 1990. As indicated in Table 4-1, there have been numerous transactions involving 241-AW-105. Most of these have been transfers of PXMSC and associated flushes. In November and December of 1994, most of the supernatant in 241-AW-105 was transferred to 241-AP-108. Since that time, 241-AW-105 has continued to receive PXMSC and flush water. Note the large number of transactions that have an unknown source. Fortunately, these volume changes are small and can be treated by simply adjusting the final supernatant volume. 
WHC-SD-WM-TI-751

Revision 0

Table 4-1. Historical Transactions for 241-AW-105. (2 Sheets) (Units are in kgal)

\begin{tabular}{|c|c|c|c|c|c|c|c|}
\hline Gain/Loss & Source & Start date & Ending date & $\begin{array}{l}\text { Beginning } \\
\text { sup volume }\end{array}$ & $\begin{array}{l}\text { Ending } \\
\text { sup } \\
\text { volume }\end{array}$ & Change & $\begin{array}{c}\text { Total tank } \\
\text { volume }\end{array}$ \\
\hline GA & Unknown & $8 / 1 / 1991$ & $8 / 31 / 1991$ & 489 & 492 & 3 & 905 \\
\hline Lo & Unknown & $9 / 1 / 1991$ & $9 / 30 / 1991$ & 492 & 489 & -3 & 902 \\
\hline Lo & Unknown & 1/ $1 / 1992$ & $1 / 31 / 1992$ & 489 & 488 & -1 & 901 \\
\hline Lo & Unknown & $3 / 1 / 1992$ & $3 / 31 / 1992$ & 488 & 486 & -2 & 899 \\
\hline GA & Unknown & 4/ $1 / 1992$ & $4 / 30 / 1992$ & 486 & 487 & 1 & 900 \\
\hline GA & PXMSC & $7 / 29 / 1992$ & $7 / 29 / 1992$ & 487 & 493 & 6 & 906 \\
\hline GA & PXMSC & $8 / 26 / 1992$ & $8 / 26 / 1992$ & 493 & 498 & 5 & 91.1 \\
\hline GA & PXMSC & $9 / 10 / 1992$ & 9/30/1992 & 498 & 522 & 24 & 935 \\
\hline GA & PXMSC & $10 / 29 / 1992$ & $10 / 30 / 1992$ & 522 & 527 & 5 & 940 \\
\hline GA & PXMSC & $11 / 14 / 1992$ & $11 / 14 / 1992$ & 527 & 532 & 5 & 945 \\
\hline GA & PXMSC & $12 / 11 / 1992$ & $12 / 30 / 1992$ & 532 & 545 & 13 & 958 \\
\hline GA & PXMSC & $1 / 16 / 1993$ & $1 / 16 / 1993$ & 545 & 550 & 5 & 963 \\
\hline GA & PXMSC & $2 / 1 / 1993$ & $2 / 27 / 1993$ & 550 & 564 & 14 & 977 \\
\hline GA & PXMSC & $3 / 6 / 1993$ & $3 / 18 / 1993$ & 564 & 573 & 9 & 986 \\
\hline LO & Unknown & 4/1/1993 & $4 / 30 / 1993$ & 573 & 570 & -3 & 983 \\
\hline LO & Unknown & 5/ $1 / 1993$ & $5 / 31 / 1993$ & 570 & 568 & -2 & 981 \\
\hline GA & PXMSC & $5 / 10 / 1993$ & $5 / 21 / 1993$ & 568 & 581 & 13 & 994 \\
\hline Lo & Unknown & $6 / 1 / 1993$ & $6 / 30 / 1993$ & 581 & 580 & -1 & 993 \\
\hline GA & PXMSC & $6 / 28 / 1993$ & $6 / 28 / 1993$ & 580 & 585 & 5 & 998 \\
\hline $\mathrm{GA}$ & PXMSC & $7 / 31 / 1993$ & $7 / 31 / 1993$ & 585 & 589 & 4 & 1002 \\
\hline GA & Unknown & $10 / 1 / 1993$ & $10 / 31 / 1993$ & 589 & 590 & 1 & 1003 \\
\hline GA & PXMSC & $10 / 20 / 1993$ & $10 / 20 / 1992$ & 590 & 597 & 7 & 1010 \\
\hline GA & PXMSC & $10 / 28 / 1993$ & $10 / 28 / 1993$ & 597 & 602 & 5 & 1015 \\
\hline GA & PXMSC & $11 / 2 / 1993$ & $11 / 18 / 1993$ & 602 & 624 & 22 & 1037 \\
\hline GA & PXMSC & $12 / 8 / 1993$ & $12 / 8 / 1993$ & 624 & 631 & 7 & 1044 \\
\hline LO & INST & $3 / 31 / 1994$ & $3 / 31 / 1994$ & 631 & 627 & -4 & 1040 \\
\hline GA & INST & $4 / 30 / 1994$ & $4 / 30 / 1994$ & 627 & 630 & 3 & 1043 \\
\hline GA & PXMSC & $6 / 17 / 1994$ & $6 / 17 / 1994$ & 630 & 635 & 5 & 1048 \\
\hline GA & PXMSC & $8 / 21 / 1994$ & $8 / 25 / 1994$ & 635 & 644 & 9 & 1057 \\
\hline GA & PXMSC & $9 / 1 / 1994$ & $9 / 20 / 1994$ & 644 & 662 & 18 & 1075 \\
\hline
\end{tabular}




\section{Revision 0}

Table 4-1. Historical Transactions for 241-AW-105. (2 Sheets)

(Units are in kgal)

\begin{tabular}{|c|c|c|c|c|c|c|c|}
\hline Gain/Loss & Source & Start date & Ending date & $\begin{array}{l}\text { Beginning } \\
\text { sup volume }\end{array}$ & $\begin{array}{l}\text { Ending } \\
\text { sup } \\
\text { volume }\end{array}$ & Change & $\begin{array}{c}\text { Total } \operatorname{tank} \\
\text { volume }\end{array}$ \\
\hline GA & PXMSC & $11 / 11 / 1994$ & $11 / 19 / 1994$ & 662 & 678 & 16 & 1091 \\
\hline GA & WATER & $11 / 11 / 1994$ & $11 / 19 / 1994$ & 678 & 680 & 2 & 1093 \\
\hline TR & $\begin{array}{c}241-A W-105 \\
241-A P-108\end{array}$ & $11 / 25 / 1994$ & $11 / 30 / 1994$ & 680 & 200 & -480 & 613 \\
\hline LO & Unknown & $12 / 1 / 1994$ & $12 / 31 / 1994$ & 291 & 289 & -2 & 611 \\
\hline TR & $\begin{array}{c}241-A W-105 \\
241-A P-108\end{array}$ & $12 / 1 / 1994$ & $12 / 3 / 1994$ & 289 & 5 & -284 & 327 \\
\hline GA & PXMSC & $12 / 5 / 1994$ & $12 / 23 / 1994$ & 5 & 20 & 15 & 342 \\
\hline GA & PXMSC & $1 / 6 / 1995$ & $1 / 31 / 1995$ & 20 & 37 & 17 & 359 \\
\hline GA & WATER & $1 / 6 / 1995$ & $1 / 31 / 1995$ & 37 & 39 & 2 & 361 \\
\hline GA & PXMSC & $2 / 3 / 1995$ & $2 / 28 / 1995$ & 39 & 61 & 22 & 383 \\
\hline GA & WATER & 2/3/1995 & $2 / 28 / 1995$ & 61 & 64 & 3 & 386 \\
\hline $\mathrm{GA}$ & WATER & $3 / 3 / 1995$ & $3 / 25 / 1995$ & 64 & 67 & 3 & 389 \\
\hline GA & PXMSC & $3 / 3 / 1995$ & $3 / 25 / 1995$ & 67 & 91 & 24 & 413 \\
\hline $\mathrm{GA}$ & WATER & 4/ 5/1995 & $4 / 25 / 1995$ & 91 & 93 & 2 & 415 \\
\hline $\mathrm{GA}$ & PXMSC & $4 / 5 / 1995$ & $4 / 25 / 1995$ & 93 & 93 & 9 & 424 \\
\hline $\mathrm{GA}$ & WATER & $5 / 1 / 1995$ & $5 / 31 / 1995$ & 102 & 105 & 3 & 427 \\
\hline $\mathrm{GA}$ & PXMSC & $5 / 1 / 1995$ & $5 / 31 / 1995$ & 105 & 151 & 46 & 473 \\
\hline LO & Unknown & $6 / 1 / 1995$ & $6 / 30 / 1995$ & 151 & 150 & -1 & 472 \\
\hline GA & PXMSC & $6 / 7 / 1995$ & $6 / 28 / 1995$ & 150 & 250 & 100 & 572 \\
\hline GA & WATER & $6 / 7 / 1995$ & $6 / 28 / 1995$ & 250 & 252 & 2 & 574 \\
\hline
\end{tabular}

NST = Instrument recalibration

PXMSC = Plutonium-uranium extraction miscellaneous wastes. 


\section{Revision 0}

Using the information in the historical transaction sheets in combination with average compositions for the waste streams added to the DSTs, one can project compositions from the date of the sample. Waste added to the DSTs come primarily from routine and decommissioning operations at the various facilities at Hanford and from saltwell liquids pumped from SSTs.

Composition data for these facility wastes come from waste profile sheets that are used to assess waste compatibility before transfer and mixing of different waste streams. These are average compositions based on historical sample data and previous waste volume submittals. These compositions are shown in Table 4-2. Composition data for saltwell liquids came from Sederburg (1995). Sederburg identifies a non-complexed saltwell liquid composition (NCSWL) and a complexed saltwell liquid composition (CSWL) with markedly different compositions. All compositions in Table 4-2 are based on mass weighted averages of a limited set of saltwell liquid data, so there is considerable uncertainty associated with them. 
Table 4-2. Facility Waste and Saltwell Liquid Composition Estimates. (2 sheets)

\begin{tabular}{|c|c|c|c|c|c|c|c|c|c|c|c|}
\hline Facility & NCSWL & CSWL & PUREX & 222 Labs & 300 Labs & B Plant & T Plant & PFP, Misc. & PFP, Labs & $100 \mathrm{~N}$ & Average DN \\
\hline COMPS & & & Misc. & & & & & & & TCO & for 106AP \\
\hline $\mathrm{Al}(\mathrm{OH}) 4-$ & $7.50 \mathrm{E}-01$ & $5.50 \mathrm{E}-01$ & & $3.70 \mathrm{E}-02$ & $1.79 \mathrm{E}-02$ & $8.04 \mathrm{E}-05$ & $1.21 \mathrm{E}-04$ & & & $2.41 \mathrm{E}-07$ & $1.93 \mathrm{E}-01$ \\
\hline $\mathrm{Ba}+2$ & & & & & & & & & & $3.85 \mathrm{E}-06$ & $6.17 \mathrm{E}-08$ \\
\hline $\mathrm{Ca}+2$ & & & & & & & & & & $3.09 \mathrm{E}-08$ & $7.74 \mathrm{E}-05$ \\
\hline $\mathrm{Cr}(\mathrm{OH}) 4$ & & & & & & & & & & $5.26 \mathrm{E}-09$ & $7.28 \mathrm{E}-05$ \\
\hline $\mathrm{FE}+3$ & & & $8.95 \mathrm{E}-04$ & $3.58 \mathrm{E}-04$ & $8.96 \mathrm{E}-06$ & & & & & $1.26 \mathrm{E}-06$ & $2.17 \mathrm{E}-05$ \\
\hline \multicolumn{12}{|l|}{$\mathrm{Hg}+2$} \\
\hline $\mathbf{K}+$ & & & & & & & & & & $3.20 \mathrm{E}-09$ & $2.68 \mathrm{E}-02$ \\
\hline \multicolumn{12}{|l|}{$\mathrm{La}+3$} \\
\hline $\mathrm{Na}+$ & $6.20 \mathrm{E}+00$ & $1.20 \mathrm{E}+01$ & $8.70 \mathrm{E}-02$ & $4.35 \mathrm{E}-01$ & $2.61 \mathrm{E}-02$ & $4.35 \mathrm{E}-01$ & $6.00 \mathrm{E}-02$ & $1.00 \mathrm{E}+00$ & $1.80 \mathrm{E}+00$ & 1.10E-08 & $1.72 \mathrm{E}+00$ \\
\hline $\mathrm{Ni}+3$ & & & & & & & & & & $4.69 \mathrm{E}-10$ & \\
\hline $\mathrm{Pb}+4$ & & & & & & & & & & $7.00 \mathrm{E}-10$ & \\
\hline $\mathrm{U} \mathbf{g} / 1$ & & & $2.00 \mathrm{E}-02$ & $1.60 \mathrm{E}-02$ & $4.00 \mathrm{E}-02$ & $1.40 \mathrm{E}-05$ & & & & & $1.60 \mathrm{E}-03$ \\
\hline $\mathrm{CO} 3-2$ & $3.20 \mathrm{E}-01$ & $7.30 \mathrm{E}-01$ & & $1.50 \mathrm{E}-02$ & $3.83 \mathrm{E}-03$ & $1.42 \mathrm{E}-02$ & $8.33 \mathrm{E}-03$ & & & $1.26 \mathrm{E}-07$ & $8.71 \mathrm{E}-02$ \\
\hline CL- & $2.11 \mathrm{E}-01$ & & $1.13 \mathrm{E}-03$ & $2.00 \mathrm{E}-02$ & $8.46 \mathrm{E}-03$ & $1.55 \mathrm{E}-03$ & $2.70 \mathrm{E}-03$ & & & $3.53 \mathrm{E}-09$ & $5.44 \mathrm{E}-02$ \\
\hline F- & $2.80 \mathrm{E}-01$ & $1.10 \mathrm{E}-01$ & & $5.00 \mathrm{E}-03$ & $5.26 \mathrm{E}-04$ & $1.61 \mathrm{E}-04$ & $1.53 \mathrm{E}-05$ & & & $1.07 \mathrm{E}-09$ & $1.17 \mathrm{E}-01$ \\
\hline SO4-2 & $4.00 \mathrm{E}-02$ & $8.80 \mathrm{E}-02$ & $2.39 \mathrm{E}-03$ & $1.00 \mathrm{E}-02$ & & $6.24 \mathrm{E}-04$ & $3.20 \mathrm{E}-04$ & & & $1.26 \mathrm{E}-09$ & $1.12 \mathrm{E}-02$ \\
\hline NO3- & $1.80 \mathrm{E}+00$ & $3.40 \mathrm{E}+00$ & $3.20 \mathrm{E}-01$ & $1.00 \mathrm{E}-01$ & $9.68 \mathrm{E}-02$ & $2.37 \mathrm{E}-02$ & & $6.00 \mathrm{E}-01$ & $1.00 \mathrm{E}-01$ & $1.61 \mathrm{E}-09$ & $4.99 \mathrm{E}-01$ \\
\hline NO2- & $1.20 \mathrm{E}+00$ & $1.90 \mathrm{E}+00$ & $1.40 \mathrm{E}-02$ & $3.00 \mathrm{E}-02$ & $8.70 \mathrm{E}-03$ & $1.50 \mathrm{E}-02$ & $1.00 \mathrm{E}-02$ & $2.00 \mathrm{E}-01$ & $4.00 \mathrm{E}-02$ & & $3.13 \mathrm{E}-01$ \\
\hline PO4-3 & $2.90 \mathrm{E}-02$ & $5.80 \mathrm{E}-02$ & $2.42 \mathrm{E}-03$ & $2.00 \mathrm{E}-02$ & $1.05 \mathrm{E}-05$ & $6.19 \mathrm{E}-03$ & $6.40 \mathrm{E}-03$ & & & $1.28 \mathrm{E}-09$ & $8.09 \mathrm{E}-03$ \\
\hline $\mathrm{OH}-$ & $2.00 \mathrm{E}+00$ & $4.30 \mathrm{E}-01$ & $4.00 \mathrm{E}-02$ & $4.00 \mathrm{E}-01$ & $1.76 \mathrm{E}-03$ & $2.34 \mathrm{E}-01$ & $3.80 \mathrm{E}-03$ & $2.00 \mathrm{E}-01$ & $1.70 \mathrm{E}+00$ & & $5.67 \mathrm{E}-01$ \\
\hline TOC $\mathrm{g} / \mathrm{L}$ & $2.90 \mathrm{E}+00$ & $4.00 \mathrm{E}+01$ & $2.30 \mathrm{E}-01$ & $3.00 \mathrm{E}+00$ & $6.00 \mathrm{E}-01$ & $1.85 \mathrm{E}-01$ & $1.00 \mathrm{E}-01$ & & & & 9.17E-01 \\
\hline
\end{tabular}


Table 4-2. Facility Waste and Saltwell Liquid Composition Estimates. (2 sheets)

\begin{tabular}{|c|c|c|c|c|c|c|c|c|c|c|c|}
\hline Facility & NCSWL & CSWL & PUREX & 222 Labs & 300 Labs & B Plant & T Plant & PFP, Misc. & PFP, Labs & $100 \mathrm{~N}$ & Average DN \\
\hline $90 \mathrm{Sr}$ & & & & $4.00 \mathrm{E}-05$ & $2.00 \mathrm{E}-04$ & $2.03 \mathrm{E}-03$ & & & & $1.82 \mathrm{E}-07$ & $6.77 \mathrm{E}-05$ \\
\hline $99 \mathrm{Tc}$ & & & & & & & & & & & $2.74 \mathrm{E}-06$ \\
\hline $237 \mathrm{~Np}$ & & & & & & & & & & & $9.16 \mathrm{E}-09$ \\
\hline $239 \mathrm{Pu}$ & & & & $4.70 \mathrm{E}-07$ & $1.24 \mathrm{E}-05$ & 2.72E-05 & $4.01 \mathrm{E}-07$ & & $1.24 \mathrm{E}-05$ & $1.42 \mathrm{E}-11$ & $1.07 \mathrm{E}-06$ \\
\hline
\end{tabular}

CSWL = Complexed saltwell liquid

DN = Dilute non-complexed

NCSWL $=$ Non-complexed saltwell liquid

PFP = Plutonium Finishing Plant

PUREX $=$ Plutonium-Uranium Extraction. 


\section{Revision 0}

Usually, there is a volume of flush water associated with each transfer. These are shown in the historical transaction sheets as water gains. For this report, the tank supernatant volumes were adjusted after each transfer of waste to account for flush volumes. A summary of historical transactions through February 1996 is provided in Table 4-3. Tanks not listed have not had any significant transfer activity.

Table 4-3. Summary of Historical Transactions for Double-Shell Tanks Supernatants. (2 sheets)

\begin{tabular}{|c|c|c|}
\hline $\begin{array}{c}\text { Transfer } \\
\text { date }\end{array}$ & $\begin{array}{l}\text { Volume } \\
\text { change } \\
\left(\mathrm{m}^{3}\right)\end{array}$ & $\begin{array}{c}\text { Source/ } \\
\text { destination }\end{array}$ \\
\hline \multicolumn{3}{|c|}{ 241-AN-101 } \\
\hline $6 / 95$ & $8.71 \mathrm{E}+02$ & NCSWL \\
\hline $11 / 95$ & $-2.31 \mathrm{E}+03$ & 241-AW-104 \\
\hline \multicolumn{3}{|c|}{ 241-AN-106 } \\
\hline 9/94 & $1.51 \mathrm{E}+03$ & Evap Bott \\
\hline $11 / 95$ & $-1.50 \mathrm{E}+03$ & Evap \\
\hline \multicolumn{3}{|c|}{ 241-AP-101 } \\
\hline $10 / 94$ & $1.07 \mathrm{E}+03$ & 241-AP-108 \\
\hline $10 / 94$ & $-1.71 E+03$ & Evap \\
\hline $1 / 95$ & $2.90 \mathrm{E}+03$ & 241-AP-108 \\
\hline $1 / 95$ & $-3.16 E+03$ & 241-AP-107 \\
\hline $8 / 95$ & $2.50 \mathrm{E}+03$ & 241-AP-105 \\
\hline \multicolumn{3}{|c|}{ 241-AP-104 } \\
\hline $7 / 95$ & $1.63 \mathrm{E}+03$ & 241-SY-102 \\
\hline $11 / 95$ & $1.31 \mathrm{E}+03$ & 241-AW-105 \\
\hline $1 / 96$ & $1.32 \mathrm{E}+02$ & B Plant \\
\hline $1 / 96$ & $1.08 \mathrm{E}+03$ & 241-AY-102 \\
\hline
\end{tabular}

\begin{tabular}{|c|c|c|}
\hline $\begin{array}{c}\text { Transfer } \\
\text { date }\end{array}$ & $\begin{array}{l}\text { Volume } \\
\text { change } \\
\left(\mathrm{m}^{3}\right)\end{array}$ & $\begin{array}{c}\text { Source/ } \\
\text { destination }\end{array}$ \\
\hline \multicolumn{3}{|c|}{ 241-AW-103 } \\
\hline $10 / 94$ & $-1.89 \mathrm{E}+03$ & 241-AP-107 \\
\hline \multicolumn{3}{|c|}{ 241-AW-104 } \\
\hline $11 / 95$ & $-4.81 \mathrm{E}+03$ & To Evap \\
\hline $11 / 95$ & $8.74 \mathrm{E}+03$ & 241-AN-101 \\
\hline \multicolumn{3}{|c|}{ 241-AW-105 } \\
\hline $11 / 94$ & $2.82 \mathrm{E}+03$ & PUREX \\
\hline $12 / 94$ & $-1.09 \mathrm{E}+04$ & 241-AP-108 \\
\hline $11 / 95$ & $4.77 \mathrm{E}+03$ & PUREX \\
\hline $11 / 95$ & $-4.96 \mathrm{E}+03$ & 241-AP-104 \\
\hline $1 / 96$ & $3.90 \mathrm{E}+03$ & PUREX \\
\hline $2 / 96$ & $-3.90 \mathrm{E}+03$ & To Evap \\
\hline \multicolumn{3}{|c|}{ 241-AY-101 } \\
\hline 7/88-5/95 & $1.26 \mathrm{E}+03$ & CSWL \\
\hline $7 / 88-5 / 95$ & $2.15 \mathrm{E}+02$ & $300 \mathrm{Lab}$ \\
\hline $12 / 88$ & $1.43 \mathrm{E}+02$ & B Plant \\
\hline $9 / 95$ & $3.21 \mathrm{E}+03$ & 241-AP-105 \\
\hline
\end{tabular}


WHC-SD-WM-TI-751

Revision 0

Table 4-3. Summary of Historical Transactions for Double-Shell Tanks Supernatants.

(2 sheets)

\begin{tabular}{|c|c|c|}
\hline $\begin{array}{l}\text { Transfer } \\
\text { date }\end{array}$ & $\begin{array}{c}\text { Volume } \\
\text { change } \\
\left(\mathrm{m}^{3}\right)\end{array}$ & $\begin{array}{c}\text { Source/ } \\
\text { destination }\end{array}$ \\
\hline $2 / 96$ & $-4.28 \mathrm{E}+03$ & To Evap \\
\hline \multicolumn{3}{|c|}{ 241-AP-105 } \\
\hline $8 / 95$ & $-2.50 \mathrm{E}+03$ & 241-AP-101 \\
\hline $9 / 95$ & $3.21 \mathrm{E}+03$ & 241-AY-101 \\
\hline $11 / 95$ & $-2.12 \mathrm{E}+03$ & To Evap \\
\hline \multicolumn{3}{|c|}{ 241-AP-107 } \\
\hline $10 / 94$ & $5.00 \mathrm{E}+02$ & 241-AW-103 \\
\hline $1 / 95$ & $3.16 \mathrm{E}+03$ & 241-AP-101 \\
\hline $6 / 95$ & $-3.66 \mathrm{E}+03$ & To Evap \\
\hline \multicolumn{3}{|c|}{ 241-AP-108 } \\
\hline $12 / 94$ & $2.89 \mathrm{E}+03$ & 241-AW-105 \\
\hline $1 / 25$ & $-2.90 \mathrm{E}+03$ & 241-AP-101 \\
\hline $5 / 95$ & $3.54 \mathrm{E}+03$ & 241-AP-106 \\
\hline $6 / 95$ & $-3.53 E+03$ & To Evap \\
\hline
\end{tabular}

\begin{tabular}{|c|c|c|}
\hline $\begin{array}{l}\text { Transfer } \\
\text { date }\end{array}$ & $\begin{array}{c}\text { Volume } \\
\text { change } \\
\left(\mathrm{m}^{3}\right)\end{array}$ & $\begin{array}{c}\text { Source/ } \\
\text { destination }\end{array}$ \\
\hline \multicolumn{3}{|c|}{ 241-AY-102 } \\
\hline $4 / 95$ & $1.29 \mathrm{E}+02$ & $222 \mathrm{Lab}$ \\
\hline $5 / 95$ & $9.08 \mathrm{E}+01$ & $300 \mathrm{Lab}$ \\
\hline Thru 5/95 & $2.83 E+03$ & Water \\
\hline $9 / 95$ & $1.15 \mathrm{E}+02$ & B Plant \\
\hline 9/95 & $2.58 \mathrm{E}+02$ & $\mathbf{T}$ Plant \\
\hline $12 / 95$ & $2.15 E+02$ & $300 \mathrm{Lab}$ \\
\hline $12 / 95$ & $7.15 \mathrm{E}+01$ & $222 \mathrm{Lab}$ \\
\hline $1 / 96$ & $1.08 \mathrm{E}+03$ & 241-AP-104 \\
\hline \multicolumn{3}{|c|}{ 241-SY-102 } \\
\hline $4 / 94$ & $1.29 \mathrm{E}+02$ & NCSWL \\
\hline $3 / 95$ & $3.15 \mathrm{E}+02$ & $222 \mathrm{Lab}$ \\
\hline $7 / 95$ & $-6.17 E+03$ & 241-AP-104 \\
\hline $12 / 95$ & $8.59 E+01$ & PFP \\
\hline
\end{tabular}


WHC-SD-WM-TI-751

Revision 0

\subsection{USING THE OPERATIONAL WASTE VOLUME PROJECTION TO ESTIMATE FUTURE COMPOSITIONS}

The OWVP (Strode 1995) is a computer model used to evaluate future DST needs. The assumptions used in the baseline case of the OWVP are based on Hanford Federal Facility Agreement and Consent Order (Tri-Party Agreement) (Ecology et al. 1994) milestones, TWRS program planning, and current operational assumptions.

The early years of the projection are modeled in more detail than later years. Specifically, the period from June 1995 through May 1997 is more detailed than later periods. The schematic in Figure 8 and the histograms in Figures 9 through 14 provided by Strode (1995) were used to project compositions for most DSTs through May 1997, and Figure 7 was used to project compositions through September 1998, with some exceptions that will be explained later. Figure 8 and Tables 3, 5, and 10 of Strode (1995) were also used to project compositions through 1998 and were critical in projecting the compositions of most DSTs from FY 1998 through FY 2002.

The OWVP also contains estimates for the amounts of flush water accompanying transfers and these estimates were included in this report. The flush volumes are dependent on the source of the waste. The OWVP generally expresses these flushes as a percentage of waste volume transferred. These assumptions are shown in Table 4-4.

Table 4-4. Operational Waste Volume Projections Flush Volume Assumptions.*

\begin{tabular}{|l|c|}
\hline \multicolumn{1}{|c|}{ Waste Source } & $\begin{array}{c}\text { Flush Volume } \\
\text { (as vol. percent of source term) }\end{array}$ \\
\hline B Plant & 0 \\
\hline Plutonium Finishing Plant & 22 \\
\hline Plutonium-Uranium Extraction & 10 \\
\hline Saltwell Liquid & 26 \\
\hline T Plant & 22 \\
\hline Cross-site Transfers & $132 \mathrm{~m}^{3} /$ year \\
\hline 100N Area & 44 \\
\hline 300 Area & 44 \\
\hline 400 Area & 44 \\
\hline 222S Labs & 22 \\
\hline
\end{tabular}

${ }^{*}$ From Strode (1995). 
WHC-SD-WM-TI-751

Revision 0

Table 4-5 summarizes the transactions that are assumed to have either taken place or will take place during the period of February 1996 through FY 2002.

Table 4-5. Summary of Assumed Future Transactions for Double-Shell Tanks Supernatants ${ }^{\mathrm{a}}$ (3 Sheets)

\begin{tabular}{|c|c|c|c|c|c|}
\hline $\begin{array}{c}\text { Tank/ } \\
\text { transfer } \\
\text { date }\end{array}$ & $\begin{array}{c}\text { Volume } \\
\text { change }\left(\mathrm{m}^{3}\right)\end{array}$ & $\begin{array}{c}\text { Source/ } \\
\text { destination }\end{array}$ & $\begin{array}{l}\text { Tank/ } \\
\text { transfer } \\
\text { date }\end{array}$ & $\begin{array}{c}\text { Volume } \\
\text { change } \\
\left(\mathrm{m}^{3}\right)\end{array}$ & $\begin{array}{c}\text { Source/ } \\
\text { destination }\end{array}$ \\
\hline \multicolumn{3}{|c|}{ 241-AN-101 } & \multicolumn{3}{|c|}{ 241-AY-101 } \\
\hline FY 1996 & $1.81 E+03$ & NCSWL & FY 1997 & $3.11 \mathrm{E}+03$ & 241-AZ-101 \\
\hline FY 1997 & $5.83 E+02$ & NCSWL & FY 00 & $1.04 E+03$ & 241-AZ-102 \\
\hline FY 1997 & $-2.40 \mathrm{E}+03$ & To Evap & \multirow{2}{*}{\multicolumn{3}{|c|}{$241-A Y-102$}} \\
\hline FY 1998 & $4.54 \mathrm{E}+02$ & 241-AZ-101 & & & \\
\hline FY 2001 & $2.03 E+03$ & 241-AZ-102 & FY03 & $6.02 \mathrm{E}+02$ & $241-C-106$ \\
\hline FY 2003 & $7.76 \mathrm{E}+02$ & 241-AY-102 & FY 03 & $-3.22 \mathrm{E}+03$ & 241-AN-101 \\
\hline \multirow{2}{*}{\multicolumn{3}{|c|}{ 241-AN-102 }} & FY 03 & $9.08 \mathrm{E}+03$ & 3 Washes \\
\hline & & & FY 03 & $-9.08 \mathrm{E}+03$ & Evap \\
\hline FY 1996 & $7.57 \mathrm{E}+01$ & Caustic & FY 03 & $2.65 \mathrm{E}+03$ & Dilution \\
\hline \multicolumn{3}{|c|}{$241-A N-106$} & \multicolumn{3}{|c|}{ 241-AZ-101 } \\
\hline FY 1997 & $1.98 \mathrm{E}+03$ & CSWL & FY 1997 & $-3.10 \mathrm{E}+03$ & 241-AY-101 \\
\hline FY 2000 & $3.39 \mathrm{E}+03$ & CSWL & FY 1998 & $4.77 \mathrm{E}+02$ & 2 Washes \\
\hline \multirow{2}{*}{\multicolumn{3}{|c|}{ 241-AN-107 }} & FY 1998 & $-4.77 \mathrm{E}+02$ & Evap \\
\hline & & & FY 1998 & $3.79 \mathrm{E}+02$ & Dilution \\
\hline FY 1996 & $1.89 \mathrm{E}+02$ & Caustic & \multicolumn{3}{|c|}{ 241-AZ-102 } \\
\hline \multicolumn{3}{|c|}{ 241-AP-101 } & FY 00 & $-1.29 E+03$ & $\mathrm{ITC}^{(\mathrm{c})}$ \\
\hline FY 1996 & $1.51 \mathrm{E}+03$ & Evap Bott & FY 00 & $-1.04 E+03$ & 241-AY-101 \\
\hline \multirow{2}{*}{\multicolumn{3}{|c|}{ 241-AP-104 }} & FY 00 & $2.11 \mathrm{E}+03$ & 2 Washes \\
\hline & & & FY 01 & $-2.11 \mathrm{E}+03$ & Evap \\
\hline FY 1996 & $3.30 \mathrm{E}+03$ & $241-S Y-102$ & FY 01 & $9.08 \mathrm{E}+02$ & Dilution \\
\hline
\end{tabular}


WHC-SD-WM-TI-751

Revision 0

Table 4-5. Summary of Assumed Future Transactions for Double-Shell Tanks Supernatants ${ }^{\mathrm{a}}$ (3 Sheets)

\begin{tabular}{|c|c|c|}
\hline $\begin{array}{l}\text { Tank/ } \\
\text { transfer } \\
\text { date }\end{array}$ & $\begin{array}{l}\text { Volume } \\
\text { change }\left(\mathrm{m}^{3}\right)\end{array}$ & $\begin{array}{c}\text { Source/ } \\
\text { destination }\end{array}$ \\
\hline FY 1997 & $-2.48 \mathrm{E}+03$ & Evap \\
\hline FY 1997 & $3.28 \mathrm{E}+03$ & 241-SY-102 \\
\hline FY 1997 & $-3.39 \mathrm{E}+03$ & Evap \\
\hline FY 1997 & $3.58 \mathrm{E}+03$ & 241-SY-102 \\
\hline FY 1997 & $-4.16 \mathrm{E}+03$ & Evap \\
\hline FY 1998 & $3.43 \mathrm{E}+03$ & 241-SY-102 \\
\hline FY 1998 & $-3.56 \mathrm{E}+03$ & Evap \\
\hline FY 1999 & $1.61 \mathrm{E}+03$ & 241-SY-102 \\
\hline FY 2002 & $2.24 \mathrm{E}+03$ & 241-SY-102 \\
\hline \multicolumn{3}{|c|}{ 241-AP-105 } \\
\hline FY 1997 & $2.43 \mathrm{E}+03$ & CSWL \\
\hline FY 1997 & $-3.86 \mathrm{E}+03$ & Evap \\
\hline $\begin{array}{c}\text { FY } 1998 \text { - } \\
99\end{array}$ & $1.58 \mathrm{E}+03$ & CSWL \\
\hline FY 1999 & $-1.98 E+03$ & Evap \\
\hline \multicolumn{3}{|c|}{ 241-AP-106 } \\
\hline FY 1996 & $3.79 \mathrm{E}+03$ & Unknown $^{(b)}$ \\
\hline \multicolumn{3}{|l|}{ FY 1996} \\
\hline \multicolumn{3}{|c|}{ 241-AP-107 } \\
\hline FY 1997 & $2.20 \mathrm{E}+03$ & Evap Bott \\
\hline FY 1999 & $1.98 \mathrm{E}+03$ & Evap Bott \\
\hline & 241-AW-104 & \\
\hline
\end{tabular}

\begin{tabular}{|c|c|c|}
\hline $\begin{array}{c}\text { Tank/ } \\
\text { transfer } \\
\text { date }\end{array}$ & $\begin{array}{c}\text { Volume } \\
\text { change } \\
\left(\mathrm{m}^{3}\right)\end{array}$ & $\begin{array}{c}\text { Source/ } \\
\text { destination }\end{array}$ \\
\hline \multicolumn{3}{|c|}{$241-\mathrm{SY}-102$} \\
\hline FY 1996 & $2.27 \mathrm{E}+01$ & PFP \\
\hline FY 1996 & $2.27 \mathrm{E}+01$ & PFP \\
\hline FY 1996 & $2.29 \mathrm{E}+03$ & NCSWL \\
\hline FY 1996 & $-3.30 \mathrm{E}+03$ & 241 -AP-104 \\
\hline FY 1997 & $9.16 \mathrm{E}+01$ & 222 S Labs \\
\hline FY 1997 & $1.40 \mathrm{E}+02$ & T Plant \\
\hline FY 1997 & $2.27 \mathrm{E}+01$ & PFP Labs \\
\hline FY 1997 & $2.27 \mathrm{E}+03$ & NCSWL \\
\hline FY 1997 & $-3.28 \mathrm{E}+03$ & 241 -AP-104 \\
\hline FY 1997 & $1.14 \mathrm{E}+02$ & 222 S Labs \\
\hline FY 1997 & $1.78 \mathrm{E}+02$ & T Plant \\
\hline FY 1997 & $2.65 \mathrm{E}+01$ & PFP Labs \\
\hline FY 1997 & $2.54 \mathrm{E}+03$ & NCSWL \\
\hline FY 1997 & $-3.58 \mathrm{E}+03$ & $241-$ AP-104 \\
\hline FY 1998 & $1.14 \mathrm{E}+02$ & 222 S Labs \\
\hline FY 1998 & $1.85 \mathrm{E}+02$ & T Plant \\
\hline FY 1998 & $2.65 \mathrm{E}+01$ & PFP Labs \\
\hline FY 1998 & $2.40 \mathrm{E}+03$ & NCSWL \\
\hline FY 1998 & $-3.43 \mathrm{E}+03$ & $241-\mathrm{AP}-104$ \\
\hline FY 1999 & $1.14 \mathrm{E}+02$ & 222 S Labs \\
\hline FY 1999 & $1.85 \mathrm{E}+02$ & T Plant \\
\hline FY 1999 & $2.65 \mathrm{E}+01$ & PFP Labs \\
\hline FY 1999 & $9.65 \mathrm{E}+02$ & NCSWL \\
\hline
\end{tabular}


WHC-SD-WM-TI-751

Revision 0

Table 4-5. Summary of Assumed Future Transactions for Double-Shell Tanks Supernatants ${ }^{\mathrm{a}}$ (3 Sheets)

\begin{tabular}{|c|c|c|}
\hline $\begin{array}{c}\text { Tank/ } \\
\text { transfer } \\
\text { date }\end{array}$ & $\begin{array}{c}\text { Volume } \\
\text { change }\left(\mathrm{m}^{3}\right)\end{array}$ & $\begin{array}{c}\text { Source/ } \\
\text { destination }\end{array}$ \\
\hline FY 1996 & $-3.63 \mathrm{E}+03$ & Evap \\
\hline FY 1996 & $5.30 \mathrm{E}+03$ & $100 \mathrm{~N}$ \\
\hline FY 1997 & $2.27 \mathrm{E}+02$ & B Plant \\
\hline FY 1997 & $2.04 \mathrm{E}+02$ & 300 Lab \\
\hline FY 1997 & $-3.31 \mathrm{E}+03$ & Evap \\
\hline FY 1998 & $2.27 \mathrm{E}+02$ & B Plant \\
\hline FY 1998 & $2.04 \mathrm{E}+02$ & 300 Lab \\
\hline FY 1999 & $2.27 \mathrm{E}+02$ & B Plant \\
\hline FY 1999 & $2.04 \mathrm{E}+02$ & 300 Lab \\
\hline FY 2000 & $2.27 \mathrm{E}+02$ & B Plant \\
\hline FY 2000 & $2.04 \mathrm{E}+02$ & 300 Lab \\
\hline FY 2001 & $1.89 \mathrm{E}+01$ & B Plant \\
\hline FY 2001 & $2.04 \mathrm{E}+02$ & 300 Lab \\
\hline FY 2002 & $1.89 \mathrm{E}+01$ & B Plant \\
\hline FY 2002 & $2.04 \mathrm{E}+02$ & 300 Lab \\
\hline
\end{tabular}

\begin{tabular}{|c|c|c|}
\hline $\begin{array}{c}\text { Tank/ } \\
\text { transfer } \\
\text { date }\end{array}$ & $\begin{array}{c}\text { Volume } \\
\text { change } \\
\left(\mathrm{m}^{3}\right)\end{array}$ & $\begin{array}{c}\text { Source/ } \\
\text { destination }\end{array}$ \\
\hline FY 1999 & $-1.61 \mathrm{E}+03$ & $241-\mathrm{AP}-104$ \\
\hline FY 2000 & $1.14 \mathrm{E}+02$ & 222 S Labs \\
\hline FY 2000 & $3.37 \mathrm{E}+02$ & T Plant \\
\hline FY 2000 & $2.65 \mathrm{E}+01$ & PFP Labs \\
\hline FY 2000 & $1.44 \mathrm{E}+02$ & NCSWL \\
\hline $\begin{array}{c}\text { FY } \\
2001-02\end{array}$ & $2.27 \mathrm{E}+02$ & 222 S Labs \\
\hline $\begin{array}{c}\text { FY } \\
2001-02\end{array}$ & $9.35 \mathrm{E}+02$ & T Plant \\
\hline $\begin{array}{c}\text { FY } \\
2001-02\end{array}$ & $4.54 \mathrm{E}+01$ & PFP Labs \\
\hline $\begin{array}{c}\text { FY } \\
2001-02\end{array}$ & $-2.24 \mathrm{E}+03$ & $241-$ AP-104 \\
\hline
\end{tabular}

$\mathrm{FY}=$ Fiscal Year

NCSWL $=$ Non-complexed saltwell liquid

PFP $=$ Plutonium Finishing Plant

a"-" denotes transfer of waste out of tank.

${ }^{b}$ A likely source is evaporator flushes and/or tank farm line flushes.

${ }^{\mathrm{c}}$ In-tank concentration. 
WHC-SD-WM-TI-751

Revision 0

\subsubsection{Assumptions not used by OWVP}

A set of assumptions different from those given in the OWVP were used for the tanks in AY and AZ Tank Farms. The OWVP assumed that the high heat sludges in 241-AY-102, 241-AZ-101, 241-AZ-102, and SST 241-C-106, that are scheduled to be retrieved into 241-AY-102, would be consolidated into one of the aging waste tanks. But because this consolidation would require modifications to the tank farm safety basis and could potentially generate unacceptable quantities of sludge wash solutions, a new set of assumptions that do not include consolidation were developed (Honeyman 1996).

These new aging waste assumptions also impact DSTs 241-AP-108 and 241-AN-101. No longer is 241-AP-108 assumed to be the storage tank for Neutralized Current Acid Waste (NCAW) supernatant from 241-AZ-102, as indicated by the OWVP. Instead, 241-AP-108 will be emptied in FY 1997 and, along with 241-AP-106, will be readied to become a contractor feed tank for Phase I privatization. Supernatants and sludge wash solutions (assumed by Honeyman to be transferred to a DST in the AN, AP, or AW farm) were assumed specifically in this report to be transferred to 241-AN-101 after its contents are sent to the 242-A Evaporator in FY 1997.

Plans to consolidate Neutralized Cladding Removal Waste (NCRW) solids in 241-AW-103 and 241-AW-105 with the solids in 241-SY-102 have not been finalized. This consolidation, if and when it occurs, will generate additional liquid volumes for DST storage. Because there is not a firm basis yet for consolidation of these solids, no modeling of consolidation for these tanks was done for this report.

Another assumption not used in the OWVP but developed for this report is the addition of caustic to 241-AN-102. Apparently, the hydroxide concentration in 241-AN-102 does not meet the minimum corrosion limit for that type of waste. It's assumed that caustic will have to be added to that tank to raise the hydroxide concentration above the corrosion limit. 


\section{WHC-SD-WM-TI-751}

Revision 0

This page intentionally left blank. 
WHC-SD-WM-TI-751

Revision 0

\subsection{SUMMARY OF TRANSACTIONS AND QUALITY OF PROJECTIONS}

The transactions, both historical and future, outlined above are summarized below for each tank. Also included in the tank summaries are the references for the tank sample data and subjective qualifiers for the projections. The projection for each tank is given a high, medium, or low qualifier. The rankings are based on the number of transactions for a particular tank, the quality of the estimated compositions, and the practicality of the transfer assumptions. For example, a high confidence level would be projected if no transactions have been completed. A summary table, Table 5-1, is included.

\section{Tank 241-AN-101}

Reference: Sutey (1993).

Supernate samples were taken on April 19, 1993. 241-AN-101 currently contains dilute non-complexed (DN) wastes and it is the receiver for NCSWL from 200 East Area. It had received $2.31 \mathrm{E}+03 \mathrm{~m}^{3}$ of saltwell liquid by June 1995. The OWVP assumed that $3.60 \mathrm{E}+03 \mathrm{~m}^{3}$ of waste would be transferred from 241-AN-101 to $241-\mathrm{AW}-104$ in the first quarter of FY 1996.

241-AN-101 will continue its role as SWL receiver and over $2.39 \mathrm{E}+03 \mathrm{~m}^{3}$ of NCSWL should be pumped in by the end of FY 1998. It's assumed that this waste will be sent to the evaporator thus enabling 241-AN-101 to receive the solutions from the washing of sludges in 241-AY-102, 241-AZ-101, and 241-AZ-102. Because of the uncertainty associated with sludge washing efficiencies, the confidence for this tank is low.

\section{Tank 241-AN-102}

\section{Reference: Herting (1994a)}

Supernate samples were taken on October 21, 1994, 241-AN-102 is designated as a complexed concentrate (CC) tank. The OWVP assumes that $241-\mathrm{AN}-102$ will remain static through FY 1998. However, the hydroxide concentration in 241-AN-102 $(0.24 M)$ is not in compliance with the minimum corrosion limit of $0.3 \mathrm{M}$. To bring the tank in compliance would require an addition of $18.9 \mathrm{~m}^{3}$ of $50 \mathrm{wt} \% \mathrm{NaOH}$. This projection assumes that $75.7 \mathrm{~m}^{3}$ of caustic will be added to bring the hydroxide concentration to $0.62 \mathrm{M}$ because this is the concentration needed to place 241-AN-102 in Envelope C.

The confidence level for 241-AN-102 is high. 


\section{WHC-SD-WM-TI-751 \\ Revision 0}

Tank 241-AN-103

Reference: Van Vleet (1993)

The sample was taken around February, 1990. 241-AN-103 currently stores doubleshell slurry (DSS). No activities are planned for 241-AN-103 before final retrieval. The confidence level for 241-AN-103 is high.

\section{Tank 241-AN-104}

Reference: Hendrickson (1994)

Starting inventories are based on estimates made for 241-AN-104 from 242-A Evaporator post-run samples taken before transfer of evaporator bottoms to $241-\mathrm{AN}-104$ and 241-AN-105. 241-AN-104 is a DSSF tank.

There have been no transfers associated with 241-AN-104 since the last sample date. No activities are planned for 241-AN-104 before final retrieval.

The confidence level for this tank is high.

Tank 241-AN-105

Reference: Hendrickson (1994)

Starting inventories are based on estimates made for 241-AN-105 from 242-A Evaporator post-run samples taken before transfer of evaporator bottoms to 241-AN-104 and 241-AN-105. 241-AN-105 is a DSSF tank.

There have been no transfers associated with 241-AN-105 since the last sample date. No activities are planned for 241-AN-105 before final retrieval.

The confidence level for this tank is high.

\section{Tank 241-AN-106}

\section{Reference: Hendrickson (1994)}

Samples were taken on April 12, 1989. Between September 9 and September 13,1994, 1.51E $+03 \mathrm{~m}^{3}$ of dilute complexed waste from 241-AW-106, the evaporator receipt tank, were transferred to 241-AN-106. Future plans include sending the partially concentrated waste back to the evaporator. Approximately six months later, 241-AN-106 is scheduled to receive CC waste from the evaporator. It was assumed that this 
waste will be a blend of waste from 241-AP-105, 241-AP-107, 241-AW-104, 241-AW-105, and the DC waste that was originally in 241-AN-106.

241-AN-106 is scheduled to be the receiver for CSWL pumped from 200 West Area tanks via 241-SY-102 through the cross-site transfer line. Because the average CSWL Na concentration is expected to be $12 M$ (Sederburg 1995), it was assumed that this waste will not be concentrated any further.

Because of the variance in saltwell liquid composition from tank to tank, there is considerable uncertainty associated with the average compositions in Sederburg. Therefore, the confidence level for this tank's projected inventory is low.

\section{Tank 241-AN-107}

Reference: Herting (1994b)

Samples were taken on May 13, 1994. 241-AN-107 is a CC tank. The only activity planned for this tank is an addition of $189 \mathrm{~m}^{3}$ of caustic to bring the hydroxide concentration within the corrosion specification.

The confidence level for this tank is high.

Tank 241-AP-101

Reference: DeLorenzo and Simpson (1994a)

Samples were taken on July 20,1993. 241-AP-101 is a DN tank. A series of transfers involving receipts of waste from 241-AP-108 and transfers to the evaporator feed tank and 241-AP-107 have already occurred. The last transfer was the receipt of DSSF from 241-AP-105 during the last quarter of FY 1995.

There are a relatively large number of transactions associated with 241-AP-101. But the compositions of the waste transferred are fairly well understood. For these reasons the confidence level for this tank is medium. 
WHC-SD-WM-TI-751

Revision 0

\section{Tank 241-AP-102}

\section{Reference: DeLorenzo and Simpson (1994a)}

The tank was last sampled on April 30, 1993 and no transfers have occurred since then. It is filled with concentrated phosphate (CP) waste. No transfers are expected to take place with this tank through FY 1997.

The confidence level for this tank is high.

Tank 241-AP-103

Reference: DeLorenzo and Simpson (1994b)

The tank was last sampled on November 25, 1991. Most of the. DN waste in 241-AP-103 was sent to the evaporator in May 1994 leaving only $102 \mathrm{~m}^{3}$ of waste behind. This tank has been designated as one of two non-aging spare tanks and will remain so through FY 1997.

The confidence level for this tank is high.

\section{Tank 241-AP-104}

\section{Reference: Van Vleet (1993)}

Samples were taken on June 1, 1988. 241-AP-104, a DN tank, will continue to receive DN wastes from 241-SY-102. This DN waste originates from facility operations including saltwell pumping of SSTs in the 200 West Area. It is assumed that 241-AP-104 will be filled and emptied with DN waste several times between now and FY 2002.

The compositions of the dilute wastes originating from facility operations in 200 West Area are based on average compositions reported in waste profile sheets. These average compositions are believed to adequately represent the waste that leaves those facilities. However, because of the fairly large number of transactions involved, the confidence level assigned to this tank is medium.

\section{Tank 241-AP-105}

Reference: DeLorenzo and Simpson (1994c)

The tank was last sampled on March 14,1993 . The waste in the tank at that time was classified as DSSF. During the last quarter of FY 1995 most of the contents of 241-AP-105, 
$2.50 \mathrm{e}+03 \mathrm{~m}^{3}$, were transferred to 241-AP-101. Supernatant from $241-\mathrm{AY}-101$ is scheduled to be transferred to 241-AP-105 before being sent to the evaporator.

241-AP-105 will become the receiver of CSWL pumped from 200 East Area after 241-AY-101, that has been the receipt tank for CSWL waste, becomes dedicated to Phase I privatization in FY 1996.

Because of the variance in saltwell liquid composition from tank to tank, there is considerable uncertainty associated with the average compositions in Sederburg. Therefore, the confidence level for this tank's projected inventory is low.

\section{Tank 241-AP-106}

Reference: DeLorenzo and Simpson (1994d)

Samples were taken on March 16 and 17, 1993. In May, 1995, most of the DN waste in 241-AP-106 was transferred to 241-AP-108 for eventual concentration in the evaporator. The OWVP then assumes that 241-AP-106 will be refilled with DN waste. that will be transferred to the evaporator feed tank in the first quarter of FY 1997. After that 241-AP-106 is assumed to receive DSSF from the evaporator. It could not be ascertained what the source of this DN addition was but it may likely come from evaporator and tank line flushes. However, by using a conservative assumption, an average composition of this waste was defined by blending all the waste streams assumed to be concentrated during that time period.

The confidence level for this tank is low.

\section{Tank 241-AP-107}

\section{Reference: DeLorenzo and Simpson (1994e)}

The tank was sampled on August 1, 1993. It contained DN waste. Historical transactions include the receipt of dilute wastes from 241-AP-101 and 241-AW-103. The contents of 241-AP-107 were transferred to the evaporator feed tank in June 1995. In FY 1997 241-AP-107 will begin receiving CC waste originating from the concentration of 200 East Area CSWL.

Because of the variance in saltwell liquid composition from tank to tank, there is considerable uncertainty associated with the average compositions in Sederburg. Therefore, the confidence level for this tank's projected inventory is low. 


\section{Tank 241-AP-108}

Reference: Miller (1994)

The last sample was taken in March, 1994. 241-AP-108 is a DN tank. Historical transaction records show a transfer of $2.89 \mathrm{E}+03 \mathrm{~m}^{3}$ from 241-AW-105. In January, 1995, $2.90 \mathrm{E}+03 \mathrm{~m}^{3}$ were sent to $241-\mathrm{AP}-101$, followed by an addition of $3.54 \mathrm{E}+03 \mathrm{~m}^{3}$ from 241-AP-106. In June, 1995, 3.53E $+03 \mathrm{~m}^{3}$ were sent to the evaporator feed tank. This waste will eventually be pumped to the evaporator feed tank. At that time, 108 will undergo preparations to become one of the feed staging tanks.

Only well characterized wastes are assumed to have resided in $241-\mathrm{AP}-108$ by the start of Phase I privatization. For this reason, the confidence level is high.

\section{Tank 241-AW-101}

Reference: Van Vleet (1993)

Samples were taken on June 28, 1990. 241-AW-101 is currently filled with DSSF. The tank is expected to remain idle through FY 2002.

The confidence level for 241-AW-101 is high.

\section{Tank 241-AW-102}

241-AW-102 is the evaporator feed tank. Because it is being filled and emptied so often, it is too difficult to project any inventory estimates.

Tank 241-AW-103

Reference: Hodgson (1995)

241-AW-103 contains Neutralized Cladding Removal Waste (NCRW). The solids in 241-AW-103 and 241-AW-105, the other NCRW tank, are considered TRU solids.

In October, 1994 about half of the supernatant in 241-AW-103 was transferred to 107AP. Future plans include the consolidation of the TRU solids in 241-AW-103, 241-AW-105, and 241-SY-102 into 241-AW-103 beginning in the first quarter of FY 1999. But because there is not a firm basis yet for consolidation of these solids, no modeling of consolidation for these tanks was done for this report.

The confidence level on the pre-consolidated composition of 241-AW-103 is high. 


\section{Revision 0}

Tank 241-AW-104

Reference: Tusler (1995)

The sample was taken November 27, 1994. 241-AW-104 is a DN tank. The waste was scheduled to be transferred to the evaporator in November, 1995. 241-AW-104 will temporarily store dilute wastes from 241-AN-101, B Plant, and the 300 Laboratory before being sent to the evaporator feed tank. This sequence of events is expected to continue through FY 2002.

Because of the uncertainty involved with the compositions of the waste added to 241-AW-104 and the number of transfers, the confidence level is medium.

\section{Tank 241-AW-105}

Reference: Schofield (1991)

241-AW-105 is a NCRW tank whose composition is based on a reconciliation of the January 1986 and July 1986 core sample data (Schofield 1991). Since 1986, 241-AW-105 has received over $871 \mathrm{~m}^{3}$ of dilute wastes from PUREX. In December 1994, 2.88E $+03 \mathrm{~m}^{3}$ were transferred to 241-AP-108. 241-AW-105 will continue to receive DN waste from PUREX and there will be periodic transfers of waste from 241-AW-105 once it fills up.

Future plans include the consolidation of the TRU solids in 241-AW-103, 241-AW-105, and 241-SY-102 into 241-AW-103 beginning in the first quarter of FY 1999. But because there is not a firm basis yet for consolidation of these solids, no modeling of consolidation for these tanks was done for this report.

The confidence level on the pre-consolidated composition of 241-AW-105 is high.

\section{Tank 241-AW-106}

241-AW-106 is the evaporator receipt tank. Because it is being filled and emptied so often, it is too difficult to project any inventory estimates.

Tank 241-AY-101

\section{Reference: Castaing (1993)}

The sample was taken on July 20,1988 . 241-AY-101 is designated as a CC tank. Since 1988, approximately $341 \mathrm{~m}^{3}$ of CSWL, $56.8 \mathrm{~m}^{3}$ of dilute laboratory waste and $37.9 \mathrm{~m}^{3}$ of DN waste from B Plant were added to 241-AY-101. Virtually all of the supernatant in 
WHC-SD-WM-TI-751

Revision 0

241-AY-101 was scheduled to be transferred to 241-AP-105 in the first quarter of FY 1996.

As part of the aging waste consolidation plan, 241-AY-101 will next receive the supernatant from 241-AZ-101 and the supernatant in 241-AZ-102 after in tank concentration has reduced the volume in that tank by approximately $1.29 \mathrm{E}+03 \mathrm{~m}^{3}$.

Waste in the AZ Farm is well characterized; thus, the confidence level for 241-AY-101 is high.

Tank 241-AY-102

Reference: Ryan (1995a)

The samples were taken in June, 1994. 241-AY-102 is a DN tank. Waste from the 222S Laboratories was added to 241-AY-102 in FY 1995. 1.07E +03 $\mathrm{m}^{3}$ were transferred to 241-AP-104 in January, 1996. As part of the Phase I privatization, the retrieved waste in 241-C-106 will be transferred to 241-AY-102. After settling, the clarified supernatant is assumed to be transferred to $241-\mathrm{AN}-101$. Afterwards, approximately $2.65 \mathrm{E}+03 \mathrm{~m}^{3}$ of a $0.1 M$ sodium hydroxide, $0.011 \mathrm{M}$ sodium nitrite solution will be added to dilute the washed sludge for future high-level waste (HLW) vitrification processing.

Projecting liquid compositions for the aging waste consolidation plan includes estimating the amount of solids that will be dissolved during water washing. The assumptions for dissolution of NCAW and 241-C-106 sludge are based on actual laboratory data for those tanks or, in the case of the caustic washing of 241-C-106, on data taken from the caustic washing of 241-C-103, a waste type similar to the waste in 241-C-106.

The aging waste tanks and the other wastes involved in aging waste consolidation have all been well characterized so the confidence level for these wastes is high. However, the process involves many transfers and assumptions on sludge washing were needed even though these assumptions rest on laboratory data. Because of these factors, the confidence level for 241-AY-102 is medium.

The confidence level for this tank is medium.

\section{Tank 241-AZ-101}

\section{Reference: Hodgson (1995)}

The samples were taken on March 3, 1995. 241-AZ-101 is a NCAW tank. The supernatant in 241-AZ-101 will be transferred to 241-AY-101 in FY 1998. The solids will then undergo a series of water washes. The wash solutions are assumed to be decanted to 241-AN-101. Finally, approximately $379 \mathrm{~m}^{3}$ of a $0.1 M$ sodium hydroxide, $0.011 M$ sodium 
nitrate solution will be added to dilute the washed sludge for future transfer for Phase I HLW vitrification processing (Honeyman 1996).

The confidence level for this tank is medium.

\section{Tank 241-AZ-102}

\section{Reference: Ryan (1995b)}

The samples were taken on February 23, 1995. As in the case of 241-AZ-101, the supernatant in 241-AZ-102 will be transferred to 241-AY-101, only this will occur in FY 2000. The solids will then undergo a series of water washes. The wash solutions are assumed to be decanted to $241-\mathrm{AN}-101$. Finally, approximately $908 \mathrm{~m}^{3}$ of a $0.1 M$ sodium hydroxide, $0.011 \mathrm{M}$ sodium nitrate solution will be added to the washed sludge for future transfer for Phase I HLW vitrification processing (Honeyman 1996).

The confidence level for this tank is medium.

\section{Tank 241-SY-101}

\section{Reference: Van Vleet (1993)}

The samples were taken on May 1, 1991. 241-SY-101 is a CC tank. No transfer activities for 241-SY-101 were assumed by OWVP before FY 2002. Two compositions are shown for 241-SY-101. The first set is similar to the other tanks--it represents supernatant data and supernatant volumes only. However, it is unlikely that the supernatant in these 241-SY-101 will ever be retrieved separate from the slurry layers. The slurry, or convective layer as it is commonly called, in 241-SY-101 is constantly being mixed to mitigate hydrogen gas generation. For these reasons, a second data set is included with a "SOL" suffix. This composition represents the soluble portion of the total inventory of 241-SY-101 and is a more likely LLW facility feed source term. Retrieval water is included in this estimate. The compositions used for the second data set were taken from the soluble compositions provided in the TWRS flowsheet (Orme 1995).

The soluble composition in Orme 1995 was generated using water wash data for 241-SY-101 taken from Herting (1994c). The wash factors from this reference probably comprise the largest uncertainty in the 241-SY-101 estimates. Even so, the confidence level for both the supernatant-only and combined soluble compositions are high because of the reproducibility of the laboratory data. 
WHC-SD-WM-TI-751

Revision 0

\section{Tank 241-SY-102}

\section{Reference: DiCenso and Winters (1995)}

The sample was taken on March 10, 1994. 241-SY-102 contains TRU solid waste primarily from the Plutonium Finishing Plant (PFP) and is considered the only PFP tank. Since the last sampling event, 241-SY-102 has received NCSWL and DN waste from the 222 Laboratory. In July 1995 much of the supernatant in 241-SY-102 was transferred to 241-AP-104. Tank 241-SY-102 will be the receiver for DN wastes from 200 West Area facilities and NCSWL/CSWL from saltwell pumping operations in 200 West Area through FY 2002.

The issue of mixing CSWL with TRU waste is being addressed since complexed wastes and TRU solids have traditionally been segregated to prevent complexing of the transuranics into solution. OWVP assumes that CSWL will be pumped into 241-SY-102 without prior retrieval of the TRU solids to meet Tri-Party Agreement milestones concerning completion of interim stabilization. This study assumes that the retrieval of TRU solids is accelerated to transfer the CSWL to 241-SY-102. The CSWL and DN wastes sent to 241-SY-102 are assumed to be transferred separately to 200 East Area tanks through the cross-site transfer line.

The confidence level for 241-SY-102 is low.

\section{Tank 241-SY-103}

\section{Reference: Hansen (1996)}

The sample was taken in September 1994. Since that time the only waste transferred to 241-SY-103 has been about $469 \mathrm{~m}^{3}$ of SWL from the 200 West Area.

As in the case of 241-SY-101, two compositions are shown, one for the "supernatant," and the other for the combined slurry. Again, retrieval water is included in the combined slurry inventory. The combined slurry composition is the more valid composition to use since a mixing pump is likely to be installed in 241-SY-103 for retrieval purposes. The slurry composition is the soluble composition found in Orme (1995). The amounts assumed to be soluble in 241-SY-103 are not based on actual laboratory data, but on basic knowledge of the chemistry of complexed waste.

The solubility assumptions for 241-SY-103-SOL are not based on laboratory data; therefore, confidence level is low. The confidence level for the supernatant only is high. 
Table 5-1. Quality of Projected Supernate Composition Estimates for Double-Shell Tanks.

\begin{tabular}{|c|c|c|c|c|}
\hline Tank & $\begin{array}{c}\text { Current } \\
\text { waste type }\end{array}$ & $\begin{array}{c}\text { Projected } \\
\text { waste type }\end{array}$ & $\begin{array}{l}\text { Projected supernatant } \\
\text { natant volume }\left(\mathrm{m}^{3}\right)\end{array}$ & $\begin{array}{l}\text { Quality of } \\
\text { projection }\end{array}$ \\
\hline 241-AN-101 & DN & $\overline{D N}$ & $3.46 \mathrm{E}+03$ & $\mathbf{L}$ \\
\hline 241-AN-102 & $\mathrm{CC}$ & $\mathrm{CC}$ & $3.82 \mathrm{E}+03$ & $\mathrm{H}$ \\
\hline 241-AN-103 & DSSF & $\overline{D S S F}$ & $3.62 \mathrm{E}+03$ & $\mathrm{H}$ \\
\hline 241-AN-104 & DSSF & DSSF & $3.02 \mathrm{E}+03$ & $\mathrm{H}$ \\
\hline $241-\mathrm{AN}-105$ & DSSF & DSSF & $4.28 \mathrm{E}+03$ & $\mathrm{H}$ \\
\hline $241-\mathrm{AN}-106$ & $\mathrm{DN}$ & $\mathrm{CC}$ & $4.01 \mathrm{E}+03$ & $\mathrm{~L}$ \\
\hline $241-\mathrm{AN}-107$ & $\overline{\mathrm{CC}}$ & $\mathrm{CC}$ & $3.68 \mathrm{E}+03$ & $\mathrm{H}$ \\
\hline 241-AP-101 & $\overline{\mathrm{DN}}$ & DSSF & $4.31 \mathrm{E}+03$ & $\mathbf{M}$ \\
\hline 241-AP-102 & CP & $\overline{C P}$ & $4.16 \mathrm{E}+03$ & $\mathrm{H}$ \\
\hline 241-AP-103 & $\mathrm{DN}$ & $\mathrm{DN}$ & $1.02 \mathrm{E}+02$ & $\mathrm{H}$ \\
\hline 241-AP-104 & DN & $\mathrm{DN}$ & $4.20 \mathrm{E}+03$ & $\mathbf{M}$ \\
\hline $241-A P-105$ & DSSF & $\overline{D C}$ & $9.99 \mathrm{E}+01$ & $\mathrm{~L}$ \\
\hline 241-AP-106 & $\mathrm{DN}$ & DSSF & $4.09 \mathrm{E}+03$ & $\mathrm{~L}$ \\
\hline 241-AP-107 & $\overline{\mathrm{DN}}$ & $\mathrm{CC}$ & $4.09 \mathrm{E}+03$ & $\mathrm{~L}$ \\
\hline 241-AP-108 & $\mathrm{DN}$ & DN & $1.01 \mathrm{E}+02$ & $\mathrm{H}$ \\
\hline 241-AW-101 & DSSF & DSSF & $3.94 \mathrm{E}+03$ & $\mathrm{H}$ \\
\hline 241-AW-102 & N/A & N/A & $\mathrm{N} / \mathrm{A}$ & N/A \\
\hline 241-AW-103 & NCRW & NCRW & $5.41 \mathrm{E}+02$ & $\mathrm{H}$ \\
\hline 241-AW-104 & $\overline{D N}$ & $\overline{\mathrm{DN}}$ & $2.27 \mathrm{E}+03$ & $\mathbf{M}$ \\
\hline 241-AW-105 & NCRW & NCRW & $9.99 \mathrm{E}+01$ & $\mathbf{H}$ \\
\hline 241-AW-106 & N/A & N/A & N/A & N/A \\
\hline $241-\mathrm{AY}-101$ & $\mathrm{CC}$ & DN & $4.20 \mathrm{E}+03$ & $\mathrm{H}$ \\
\hline $241-\mathrm{AY}-102$ & $\mathrm{DN}$ & DN & $2.90 \mathrm{E}+03$ & $\mathbf{M}$ \\
\hline 241-AZ-101 & $\mathrm{DN}$ & DN & $6.25 \mathrm{E}+02$ & $\mathrm{M}$ \\
\hline 241-AZ-102 & DN & DN & $1.16 \mathrm{E}+03$ & $\mathbf{M}$ \\
\hline $241-S Y-101$ & $\mathrm{CC}$ & $\overline{\mathrm{CC}}$ & $6.81 \mathrm{E}+01$ & $\mathrm{H}$ \\
\hline $241-S Y-102$ & DN & $\mathrm{DN}$ & $9.99 \mathrm{E}+01$ & $\mathbf{L}$ \\
\hline $241-S Y-103$ & $\mathrm{CC}$ & $\mathrm{CC}$ & $6.43 \mathrm{E}+02$ & $\mathrm{H}$ \\
\hline 241-SY-101-SOL & $\mathrm{CC}$ & $\mathrm{CC}$ & $7.38 \mathrm{E}+03$ & $\mathrm{H}$ \\
\hline 241-SY-103-SOL & $\mathrm{CC}$ & $\mathrm{CC}$ & $4.43 E+03$ & $\mathbf{L}$ \\
\hline
\end{tabular}

$\mathrm{CC}=$ Complexant concentrate

$\mathrm{CP}=$ Concentrated Phosphate

$\mathrm{DN}=$ Dilute non-complexed

DSSF $=$ Double-shell slurry feed

NCRW $=$ Neutralized cladding removal waste. 


\section{WHC-SD-WM-TI-751}

Revision 0

This page intentionally left blank. 
WHC-SD-WM-TI-751

Revision 0

\subsection{REFERENCES}

Carpenter, K. E., 1995, Tank Waste Remediation System Functions and Requirements Document, WHC-SD-WM-FRD-020, Rev. B, Draft, Westinghouse Hanford Company, Richland, Washington.

Castaing, B. A., 1994, 101-AY, 102-AY, \& 106-C Data Compendium, WHC-SD-WM-TI-578, Rev. 1, Westinghouse Hanford Company, Richland, Washington.

Certa, P. J., C. M. McConville, L. W. Shelton, and E. J. Slaathaug, 1996a, Preliminary Low-Level Waste Feed Staging Plan, WHC-SD-WM-RPT-210, Rev. 0, Westinghouse Hanford Company, Richland, Washington.

Certa, P. J., W. H. Grams, C. M. McConville, L. W. Shelton, and E. J. Slaathaug, 1996b, Low-Level Waste Feed Staging Plan, WHC-SD-WM-RPT-224, Rev. 0, Westinghouse Hanford Company, Richland, Washington.

DeLorenzo, D. S. and Simpson, B. C., 1994a, Tank Characterization Report for DoubleShell Tank 241-AP-101, WHC-SD-WM-ER-357, Westinghouse Hanford Company, Richland, Washington.

DeLorenzo, D. S. and Simpson, B. C., 1994b, Tank Characterization Report for DoubleShell Tank 241-AP-103, WHC-SD-WM-ER-359, Rev. 0, Westinghouse Hanford Company, Richland, Washington.

DeLorenzo, D. S, and Simpson, B. C., 1994c, Tank Characterization Report for DoubleShell Tank 241-AP-105, WHC-SD-WM-ER-360, Westinghouse Hanford Company, Richland, Washington.

DeLorenzo, D. S. and Simpson, B. C., 1994d, Tank Characterization Report for DoubleShell Tank 241-AP-106, WHC-SD-WM-ER-361, Westinghouse Hanford Company, Richland, Washington.

DeLorenzo, D. S. and Simpson, B. C., 1994e, Tank Characterization Report for DoubleShell Tank 241-AP-107, WHC-SD-WM-ER-362, Westinghouse Hanford Company, Richland, Washington.

DiCenso, A. T. and W. I. Winters, 1995, Tank Characterization Report for Double-Shell Tank 241-SY-102, WHC-SD-EM-ER-366, Rev. 0, Westinghouse Hanford Company, Richland, Washington.

DOE-RL, 1995, Draft, TWRS Privatization Request for Proposals, Solicitation Number DE-RP06-96RL13308, U.S. Department of Energy-Richland Operations Office, Richland, Washington. 


\section{Revision 0}

Ecology, EPA, and DOE, 1994, Hanford Federal Facility Agreement and consent Order, As Amended, Washington State Department of Ecology, U.S. Environmental Protection Agency, and U.S. Department of Energy, Olympia, Washington.

Hansen, D. R., 1996, Tank Characterization Report for Double-Shell Tank 24I-SY-103, WHC-SD-WM-ER-471, Rev. 0, Westinghouse Hanford Company, Richland, Washington.

Hendrickson, D. W., 1994, Grout Treatment Facility Waste Feed Projections, WHC-SD-WM-TI-528, Rev. 1, Westinghouse Hanford Company, Richland, Washington.

Herting, D. L., 1994a, Characterization of Supernate Samples from Tank 102-AN, Internal memo to J. M. Jones, (December 28, 1994), Westinghouse Hanford Company, Richland, Washington.

Herting, D. L., 1994b, Characterization of Sludge Samples form Tank 241-AN-107, Internal Memo to K. G. Carothers,(July 21, 1994), Westinghouse Hanford Company, Richland, Washington.

Herting, D. L., 1994c, Chemical Composition of Tank 10I-SY Solids, Internal Memo to G. D. Johnson, (July 11, 1994), Westinghouse Hanford Company, Richland, Washington.

Hodgson, K. M., 1995a, Tank Characterization Report for Double-Shell Tank 24I-AZ-10I, Westinghouse Hanford Company, WHC-SD-WM-ER-410, Rev. 0, Richland, Washington.

Hodgson, K. M., 1995b, Tank Characterization Report for Double-Shell Tank 241-AW-103, WHC-SD-WM-ER-455, Rev. 0, Westinghouse Hanford Company, Richland, Washington.

Honeyman, J. O., 1996, Submittal of the TWRS Disposal Program Assumptions for the Operational Waste Volume Projection, Internal Memo from J.O. Honeyman to W. B. Barton, (March 1, 1996), Westinghouse Hanford Company, Richland, Washington.

Miller, G. L., 1994, Analysis and Characterization of Double-Shell Tank 241-AP-108, WHC-SD-WM-DP-065, Westinghouse Hanford Company, Richland, Washington.

Orme, R. M., 1995, TWRS Process Flowsheet, WHC-SD-WM-TI-613, Rev. 1, Westinghouse Hanford Company, Richland, Washington.

ORNL, 1995, Integrated Data Base Report-1994: U.S. Spent Nuclear Fuel and Radioactive Waste Inventories, Projections, and Characteristics, DOE/RW-0006, Rev. 11, Oak Ridge National Laboratory, Oak Ridge, Tennessee. 
Ryan, G. W., 1995a, Tank Characterization Report for Double-Shell Tank 241-AY-102, WHC-SD-WM-ER-454, Rev. 0, Westinghouse Hanford Company, Richland, Washington.

Ryan, G. W., 1995b, Tank Characterization Report for Double-Shell Tank 241-AZ-102, WHC-SD-WM-ER-411, Rev. 0, Westinghouse Hanford Company, Richland, Washington.

Schofield, J. S., 1991, Estimation of Neutralized Current Acid Waste And Neutralized Cladding Removal Waste Constituents, Internal Memo to S. L. Barker, (July 18, 1991), Westinghouse Hanford Company, Richland, Washington.

Sederburg, J. P., 1995, Waste Volume Reduction Factors for Potential 242-A Evaporator Feed, WHC-SD-WM-TI-690, Rev. 0, Westinghouse Hanford Company, Richland, Washington.

Strode, J. N., 1995, Operational Waste Volume Projection, WHC-SD-WM-ER-029, Rev. 21, Westinghouse Hanford Company, Richland, Washington.

Sutey, M. J., 1993, Waste Compatibility Assessment of Tank 241-AN-101 with Tanks 241-BX-110 and 241-BX-111, Internal Memo to S. D. Godfrey (August 3, 1993), Westinghouse Hanford Company, Richland, Washington.

Tusler, L. A., 1995, Tank Characterization Report for Double-Shell Tank 241-AW-104, WHC-SD-WM-ER-453, Rev. 0, Westinghouse Hanford Company, Richland, Washington.

Van Vleet, R. J., 1993, WHC-SD-TI-543 Rev. 0, Radionuclide and Chemical Inventories for the Double-Shell Tanks, Westinghouse Hanford Company, Richland, Washington.

WHC, 1995, Tank Waste Remediation System Functions and Requirements Document, WHC-SD-WM-FRD-020, Draft Rev. E, Westinghouse Hanford Company, Richland, Washington. 
WHC-SD-WM-TI-751

Revision 0

This page intentionally left blank. 
DISTRIBUTION SHEET

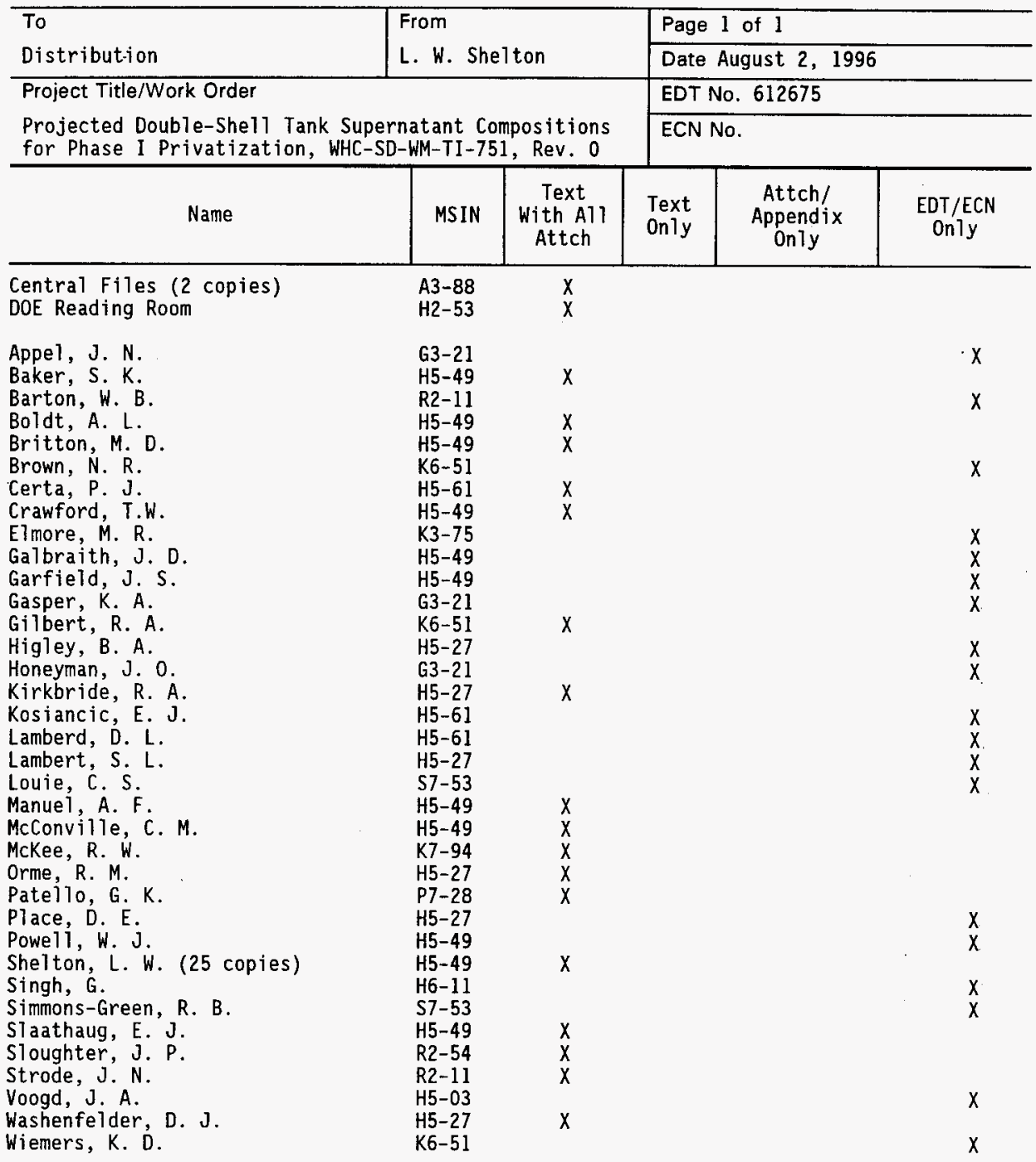

